\title{
Heterogeneity of leukemia-initiating capacity of chronic myelogenous leukemia stem cells
}

\author{
Bin Zhang, ${ }^{1}$ Ling Li, ${ }^{1}$ Yinwei Ho, ${ }^{1}$ Min Li, ${ }^{2}$ Guido Marcucci, ${ }^{1}$ Wei Tong, ${ }^{3}$ and Ravi Bhatia ${ }^{4}$ \\ 'Division of Hematopoietic Stem Cell and Leukemia Research, Cehr Family Center for Leukemia Research, and ²Division of Biostatistics, City of Hope National Medical Center, Duarte, California, USA. \\ ${ }^{3}$ Department of Pediatrics, Children's Hospital of Philadelphia, Philadelphia, Pennsylvania, USA. ${ }^{4}$ Division of Hematology and Oncology, Department of Medicine, University of Alabama Birmingham, \\ Birmingham, Alabama, USA.
}

\begin{abstract}
Chronic myelogenous leukemia (CML) results from transformation of a long-term hematopoietic stem cell (LTHSC) by expression of the BCR-ABL fusion gene. However, BCR-ABL-expressing LTHSCs are heterogeneous in their capacity as leukemic stem cells (LSCs). Although discrepancies in proliferative, self-renewal, and differentiation properties of normal LTHSCs are being increasingly recognized, the mechanisms underlying heterogeneity of leukemic LTHSCs are poorly understood. Using a CML mouse model, we identified gene expression differences between leukemic and nonleukemic LTHSCs. Expression of the thrombopoietin (THPO) receptor MPL was elevated in leukemic LTHSC populations. Compared with LTHSCs with low MPL expression, LTHSCs with high MPL expression showed enhanced JAK/STAT signaling and proliferation in response to THPO in vitro and increased leukemogenic capacity in vivo. Although both $\mathrm{G}_{0}$ and $\mathrm{S}$ phase subpopulations were increased in LTHSCs with high MPL expression, LSC capacity was restricted to quiescent cells. Inhibition of MPL expression in CML LTHSCs reduced THPO-induced JAK/STAT signaling and leukemogenic potential. These same phenotypes were also present in LTHSCs from patients with CML, and patient LTHSCs with high MPL expression had reduced sensitivity to BCR-ABL tyrosine kinase inhibitor treatment but increased sensitivity to JAK inhibitors. Together, our studies identify MPL expression levels as a key determinant of heterogeneous leukemia-initiating capacity and drug sensitivity of CML LTHSCs and suggest that high MPL-expressing CML stem cells are potential targets for therapy.
\end{abstract}

\section{Introduction}

Chronic myelogenous leukemia (CML) is a lethal hematological disorder originating from a small population of leukemia stem cells (LSCs). CML cells are characterized by the presence of the $B C R-A B L$ oncogene, which plays a critical role in hematopoietic stem cell (HSC) transformation (1). HSC transformation results in a vast expansion of malignant myeloid cells, which retain differentiating capacity. Leukemic cells are prone to acquire additional genetic abnormalities over time, resulting in disease progression from an initial chronic phase to an advanced accelerated phase and blast crisis (2).

Inhibition of BCR-ABL activity with tyrosine kinase inhibitors (TKIs) is remarkably effective in inducing remission and prolonging survival in patients with CML. However, CML LSCs usually persist in patients achieving remissions following TKI treatment and frequently result in leukemia relapse on discontinuation of TKI treatment (3). As a result, most patients require continued TKI treatment to prevent relapse. However, small subsets of patients with CML that attain sustained deep remissions maintain longterm remission after discontinuing TKI treatment (4). Patients maintaining treatment-free remissions continue to demonstrate low levels of $\mathrm{BCR}-\mathrm{ABL}^{+}$cells when analyzed using sensitive assays, indicating persistence of $\mathrm{BCR}-\mathrm{ABL}^{+}$stem cells (5). The lack of leu-

Conflict of interest: The authors have declared that no conflict of interest exists. Submitted: September 25, 2014; Accepted: January 4, 2016.

Reference information: / Clin Invest. 2016;126(3):975-991. doi:10.1172/JCI79196. kemia recurrence in these patients suggests limited potential of residual CML long-term HSCs (LTHSCs) to regenerate leukemia and could be explained by heterogeneity in leukemogenic potential of BCR-ABL ${ }^{+}$LTHSCs, in conjunction with restriction of leukemic LTHSC growth by microenvironmental and/or immune factors.

Clonal heterogeneity of proliferative, self-renewal, and differentiation properties of normal HSCs has been recognized $(6,7)$. However, heterogeneity of function of well-defined, oncogeneexpressing LSCs is less well studied. Previous studies have indicated that CML LSCs have a phenotype that is similar to that of normal LTHSCs (8). As with normal human LTHSCs, LSCs from patients with $\mathrm{CML}$ share the $\mathrm{CD} 34^{+} \mathrm{CD} 38^{-} \mathrm{CD} 90^{+}$phenotype (8). CML LSCs demonstrate enhanced proliferation, reduced apoptosis, and increased differentiation in vitro compared with normal LTHSCs. Although human CML LSCs regenerate leukemic cells when transplanted into immunodeficient mice, engraftment levels are low and recipient mice do not develop leukemia, limiting the utility of this approach to study in vivo CML LSC growth. We therefore used an inducible transgenic mouse model of CML in which the $B C R-A B L$ gene is expressed under the control of a tetracycline-regulated $3^{\prime}$ enhancer of the stem cell leukemia $(S C L)$ gene to study CML LSCs in greater detail $(9,10)$. In this model, BCR-ABL expression in HSCs following tetracycline withdrawal results in a chronic myeloproliferative disorder that resembles chronic phase CML. CML cells with long-term repopulating and leukemia-initiating capacity demonstrate a lineage-Sca- ${ }^{+} \mathrm{c}-\mathrm{Kit}^{+}$ (LSK) $\mathrm{Flt3}^{-} \mathrm{CD} 150^{+} \mathrm{CD} 48^{-}$phenotype, similar to that of normal 
A

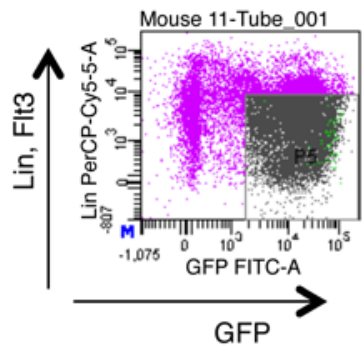

B

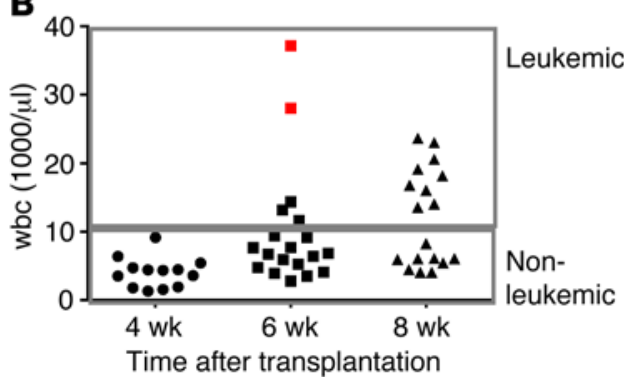

E

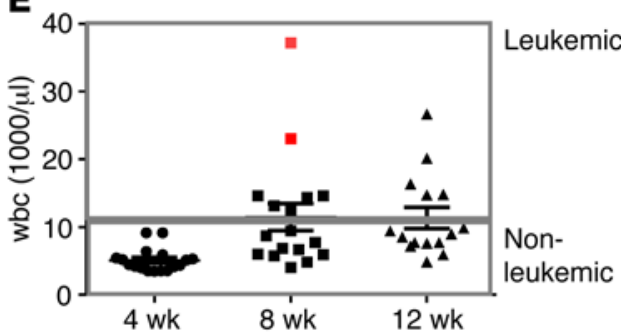

Time after transplantation

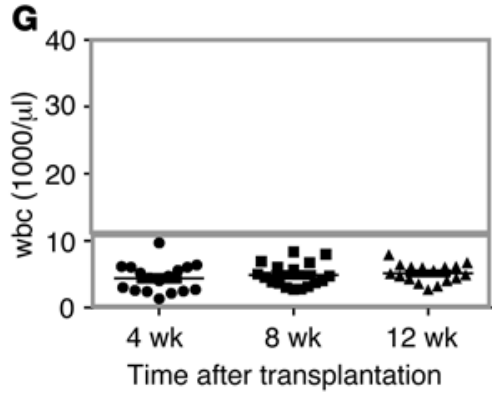

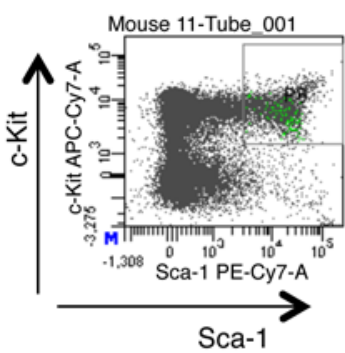

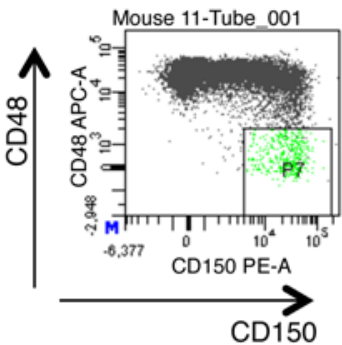

C

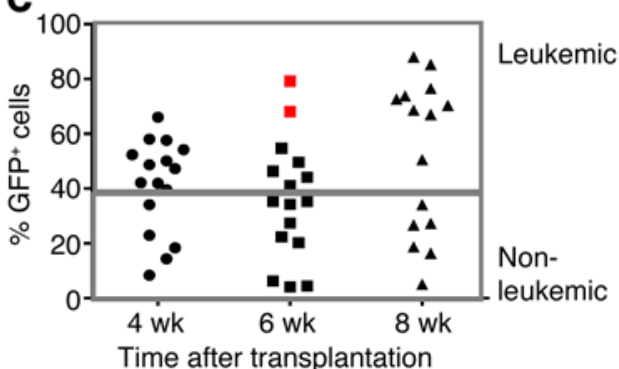

$\mathbf{F}$

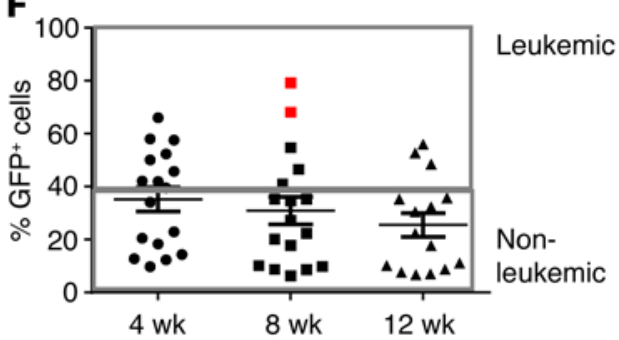

Time after transplantation

H

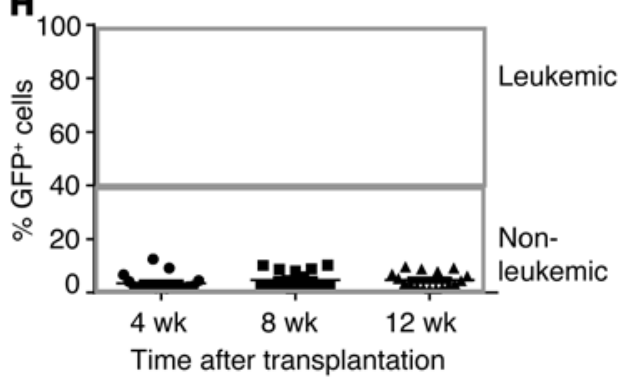

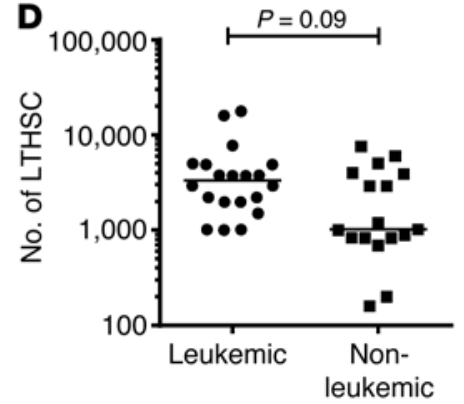

I

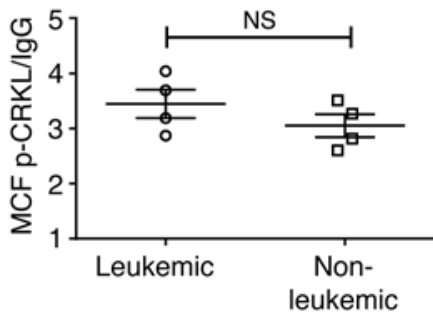

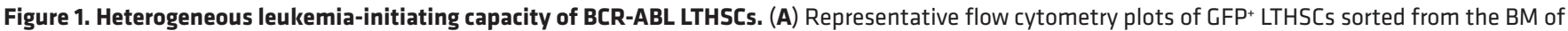
control and BCR-ABL-expressing mice. (B and C) 200 GFP+ LTHSCs per mouse were transplanted into recipient mice $(n=20)$, and wbc count and engraftment of GFP+ donor cells in PB were followed. (D) GFP+ LTHSCs were selected from primary leukemic and nonleukemic recipient mice and transplanted into secondary recipient mice (200 cells per mouse, $n=17$ ). wbc count and GFP+ cells in the PB of secondary recipient mice was monitored every 4 weeks after transplantation. (E and F) Recipients transplanted with GFP+ LTHSCs from leukemic mice. (G and $\mathbf{H})$ Recipients transplanted with GFP+ LTHSCs from nonleukemic mice. Red spots represent the mice that were sick and euthanized or found dead. (I) LTHSCs were selected from BM of nonleukemic and leukemic recipient mice at 8 weeks after transplantation, and p-CRKL expression was analyzed by flow cytometry. Results represent mean \pm SEM. Student's $t$ test was used to assess significance.

LTHSCs $(11,12)$. However, the frequency of BCR-ABL ${ }^{+}$cells with LTHSC phenotype with leukemia-initiating capacity is significantly lower than that of those with long-term engrafting capacity (11). This observation suggests that CML LTHSCs may be heterogeneous and that only a small proportion of LTHSCs with longterm engrafting capacity have leukemia-initiating capacity.

In the current study, we analyzed gene expression patterns associated with the leukemia-initiating capacity of BCR-ABLexpressing LTHSCs using the transgenic BCR-ABL mouse model of CML. The results led us to evaluate the relationship of expression of the thrombopoietin (THPO) receptor MPL with leukemia- initiating potential of BCR-ABL-expressing LTHSCs and the direct contribution of MPL signaling to the leukemogenic capacity of BCR-ABL ${ }^{+}$LTHSCs. Finally, we evaluated the relationship of MPL expression with proliferative and regenerative capacity of human CML LTHSCs.

\section{Results}

Heterogeneity in leukemia-initiating capacity of CML LTHSCs. Our previous studies using the SCL-tTA/BCR-ABL mouse model of $\mathrm{CML}$ indicate that long-term repopulation and leukemia-initiating capacity after transplantation is restricted to cells with the LTHSC 
phenotype (LSK Flt3 ${ }^{-} \mathrm{CD} 150^{+} \mathrm{CD} 48^{-}$) (11). Limiting dilution studies showed that the frequency of cells with LTHSC phenotype with long-term engraftment capacity was approximately 10-fold higher than that of those with leukemia-initiating capacity, suggesting that only a subfraction of long-term engrafting cells have LSC capacity (11). To further evaluate heterogeneity in LSC potential, SCL-tTA/BCR-ABL mice were crossed with GFP-expressing mice to allow tracking of donor cells, and $200 \mathrm{GFP}^{+}$donor LTHSCs per mouse were transplanted into a cohort of congenic FVBN mice. Recipient mice were followed for engraftment of $\mathrm{GFP}^{+}$cells and development of CML. We observed that primary recipients demonstrated variable levels of donor cell engraftment and leukocytosis. Since leukemia development in this mouse model usually takes place within 8 weeks, and mice that develop leukemia usually become sick or die within this period of time, for practical reasons, mice were followed for up to 8 weeks after transplant in this experiment (11). Eleven of twenty recipient mice developed leukocytosis characteristic of CML associated with high levels of donor cell engraftment ("leukemic mice"), whereas nine mice showed long-term engraftment of $\mathrm{GFP}^{+}$cells without development of leukocytosis ("nonleukemic mice") (Figure 1, A-C). There was a significant correlation between high wbc count in recipient mice and high $\mathrm{GFP}^{+}$donor cell engraftment $\left(r^{2}=0.74, P<0.001\right)$. $\mathrm{GFP}^{+}$LTHSCs were selected from primary recipients at 8 weeks after transplant and transplanted into secondary recipient mice at 200 cells per mouse. There was a trend toward a higher number of $\mathrm{GFP}^{+}$LTHSCs being obtained from leukemic mice compared with that from nonleukemic mice (Figure 1D). Seven of seventeen mice receiving cells from leukemic mice developed CML after the second transplant, whereas none of the mice receiving cells from nonleukemic mice developed CML (Figure 1, E-H). These observations suggest that the distinction between LTHSCs with leukemogenic versus nonleukemogenic capacity is maintained with serial transplantation. LTHSCs from both nonleukemic and leukemic recipient mice showed increased p-CRKL expression, indicating evidence for BCR-ABL activity in both populations (Figure 1I).

Differential gene expression in leukemogenic LTHSCs. LTHSCs isolated from leukemic and nonleukemic primary recipients were analyzed for expression of a panel of HSC regulatory genes by multiplex quantitative RT-PCR (Q-RT-PCR). Genes were selected to reflect regulatory mechanisms of known importance, including Wnt signaling (Fzd7, Ppard, Ccnd1), Hedgehog signaling (Gli1, Gli2, Ptchd1), Notch signaling (Hes1), and SIRT1 and p53 signaling activity (Sirt1, p53, p16, p21, Mdm2, Bax, Puma, Noxa, necdin, $G f i 1, D R 5)$. We also selected other stem cell regulatory genes $(M y c$, Bmi1, Pten, Foxo1), genes involved in niche interactions (Cxcr4, Tie2, Mpl, Cdh2, Itga4, Cd47), $B C R-A B L$, and housekeeping genes (actin, B2m, Hprt1). Using hierarchical clustering, LTHSCs from leukemic mice clustered separately from those from nonleukemic mice (Figure 2A). We observed significant differences in expression of $M p l(P<0.001), M y c(P<0.001), C d 47(P<0.001)$, Pten $(P<0.01)$, Sirt1 $(P<0.001)$, Ptch1 $(P<0.001)$, and Tie $2(P<0.001)$ (Figure $2 \mathrm{~B}) . B C R-A B L$ expression was not significantly different in LTHSCs from mice with leukemia compared with that in LTHSCs without leukemia. Given the importance of THPO/MPL interactions in maintenance of normal HSCs, we focused further attention on the relationship of increased $M p l$ expression with leukemogenic capacity of BCR-ABL ${ }^{+}$LTHSCs (13-16). Expression of $M p l$ showed a significant correlation with expression of $M y c$, Cd47, Ptch1, Sirt1, and Tie2 in BCR-ABL ${ }^{+}$LTHSCs but not with expression of Pten or BCR-ABL (Supplemental Figure 1; supplemental material available online with this article; doi:10.1172/ JCI79196DS1). Flow cytometry analysis of MPL expression in CML and normal stem cells revealed that, within LSK cells, the highest levels of MPL expression were seen in LTHSCs (Figure 2, C and D). CML LTHSCs expressed lower levels of MPL compared with normal LTHSCs (Figure 2D). In addition, Q-RT-PCR analysis showed reduced $M p l$ mRNA expression in CML LTHSCs compared with that in normal LTHSCs (Figure 2E).

Recognizing that additional mechanisms, beyond those studied here, could also contribute to the functional heterogeneity of LSCs in CML, we compared global gene expression in LTHSCs from leukemic and nonleukemic mice using RNA sequencing (RNA-Seq). As expected, this analysis identified additional categories of genes that were differentially expressed between leukemic and nonleukemic LTHSCs. Analysis of enriched gene groups using DAVID identified significant and prominent enrichment of immune response-related genes in nonleukemic LTHSCs (Supplemental Table 1). Gene set enrichment analysis (GSEA) further confirmed that several gene sets related to immune response were enriched in nonleukemic LTHSCs (Supplemental Table 2). These observations support an association between responsiveness to immune response and the leukemogenic potential of BCR-ABLexpressing LTHSCs (Supplemental Table 1). GSEA also indicated enrichment of HSC maturation-related genes and downregulation of MYC target genes in nonleukemic LTHSCs (Supplemental Table 2). Interestingly, consistent with recent reports that PPAR $\gamma$ agonists can deplete quiescent CML LSCs, PPAR $\gamma$-related gene sets (LAL_KO_3MO_UP, LAL_KO_6MO_UP) were also observed in nonleukemic LTHSCs (17).

Enhanced leukemogenic capacity of LTHSCs selected for high $M P L$ expression. We next used flow cytometry to select BM LTHSCs with high and low levels of cell surface MPL expression (highest and lowest 20\%; MPL ${ }^{\text {hi }}$ and $\mathrm{MPL}^{\text {lo }}$, respectively) from BCR-ABL and control mice (Figure 3A). Differential $M p l$ mRNA expression was confirmed by Q-RT-PCR (Figure 3B). BCR-ABL and normal MPL $^{\text {hi }}$ LTHSCs generated significantly higher cell numbers in suspension culture (Supplemental Figure 2A) and increased colony numbers in colony-forming cell (CFC) assays (Supplemental Figure 2B) when cultured with THPO compared with $\mathrm{MPL}^{\text {lo }}$ LTHSCs. In addition, BCR-ABL MPL ${ }^{\text {hi }}$ LTHSCs also showed markedly increased cell expansion and CFC potential compared with normal MPL ${ }^{\text {hi }}$ LTHSCs. We did not observe significant differences in apoptosis between MPL ${ }^{\text {hi }}$ and MPL ${ }^{\text {lo }}$ LTHSCs from BCR-ABL and normal mice (Supplemental Figure 2C). To determine in vivo regenerative capacity, BCR-ABL $M P L^{\text {hi }}$ and MPL $^{\text {lo }}$ LTHSCs (CD45.1, 100 cells per mouse) were transplanted into congenic recipients (CD45.2) and followed for engraftment and leukemia development. Seven of sixteen mice that received MPL $^{\text {hi }}$ LTHSCs developed leukemia after transplantation compared with one of seventeen mice that received MPL ${ }^{\text {lo }}$ LTHSCs, indicating an increased leukemogenic capacity of MPL hi LTHSCs (Figure 3, C and D). MPL ${ }^{\text {hi }}$ LTHSCs generated significantly higher donor cell engraftment compared with MPL ${ }^{\text {lo }}$ LTHSCs. Transplan- 
A
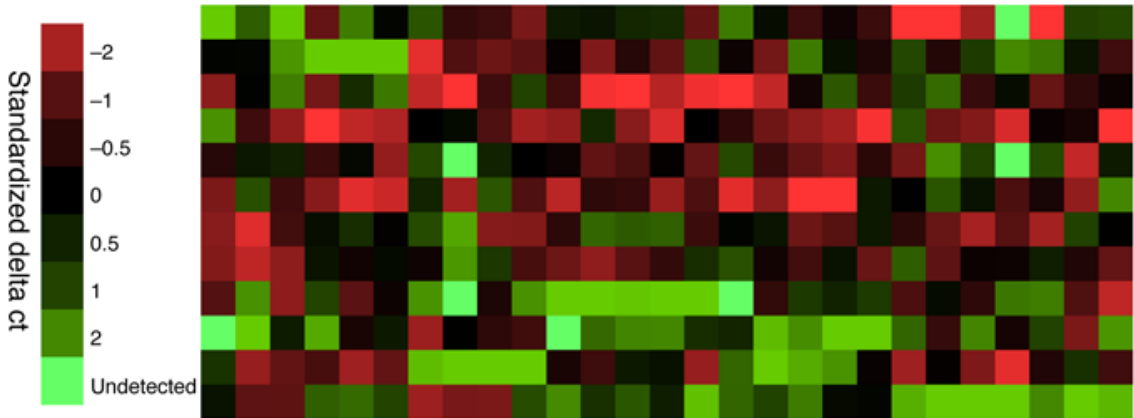

CML 2-NEP

CML 12

CML 10

CML 2-LEP

CML 4-LEP

CML 13

CML 4-BEP

Non-CML 3-NEP

Non-CML 5-NEP

Non-CML 5-REP

Non-CML

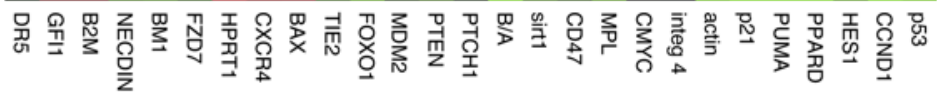

B
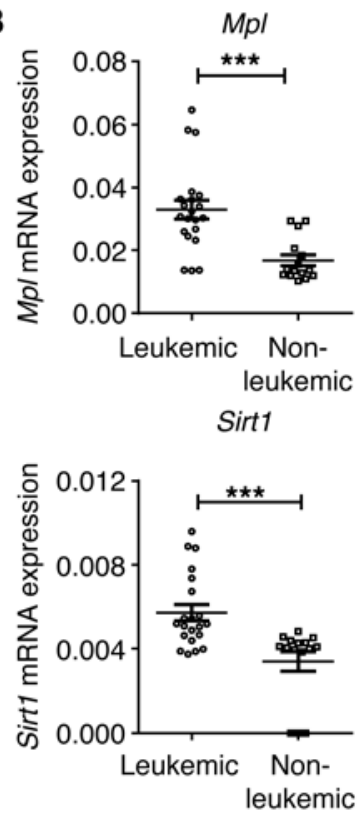

C

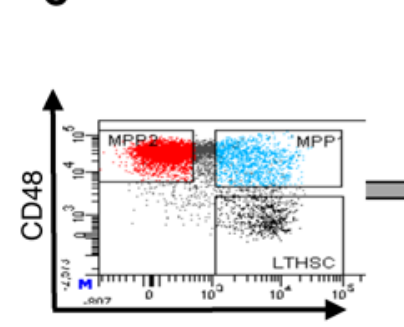

CD150

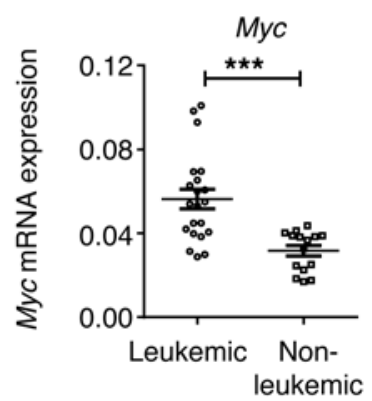

Ptch1

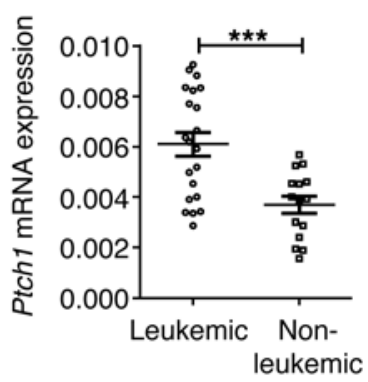

D

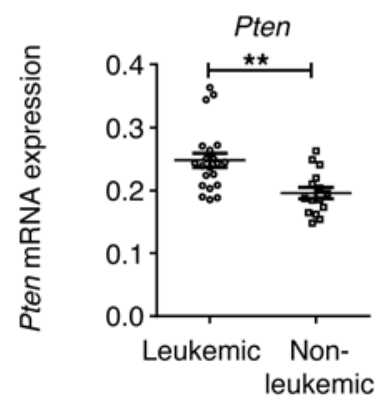

Tie2

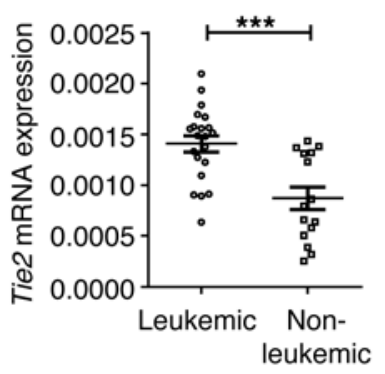

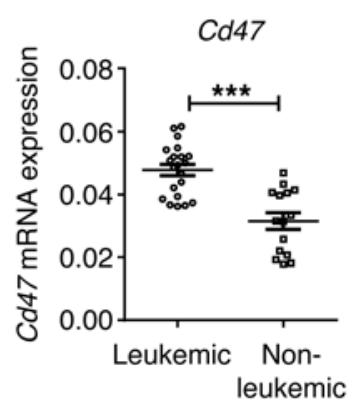

$B C R-A B L$

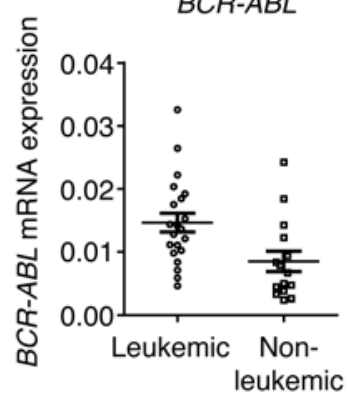

E
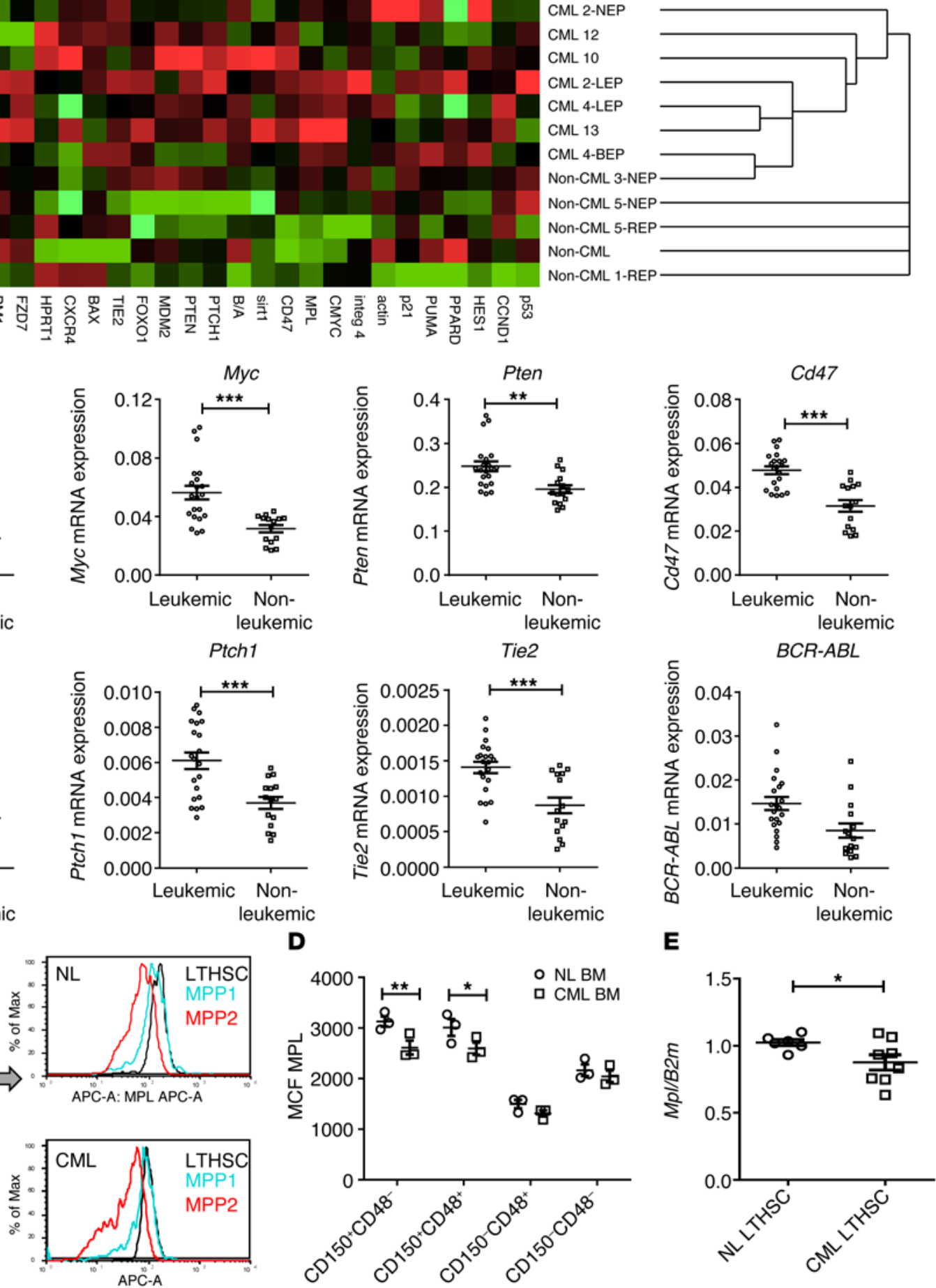

Figure 2. Gene expression patterns in LTHSCs from leukemic and nonleukemic mice. (A) Heatmap showing hierarchical clustering of expression of a panel of HSC regulatory genes in LTHSCs isolated from leukemic and nonleukemic primary recipients (as shown in Figure 1) analyzed by multiplex Q-RT-PCR. (B) Expression of specific genes in LTHSCs isolated from leukemic and nonleukemic primary recipients. (C) Representative flow cytometry plots of $\mathrm{Mpl}$ expression in CML and normal LSK subpopulations and (D) combined results. MCF, median channel fluorescence. (E) Mpl mRNA expression in normal and CML LTHSCs. Results represent mean $\pm \mathrm{SEM}$. ${ }^{*} P<0.05 ;{ }^{* *} P<0.01 ;{ }^{* *} P<0.001$. 2-way ANOVA was used to assess significance.

tation of normal MPL hi LTHSCs (CD45.1) into normal recipients (CD45.2, 50 cells per mouse) also resulted in significantly higher long-term engraftment of donor cells compared with transplantation of MPL ${ }^{\text {lo }}$ LTHSCs (Figure 3E). Analysis of donor LTHSC engraftment in $\mathrm{BM}$ of mice receiving $\mathrm{MPL}^{\text {hi }}$ and $M P L^{\text {lo }} \mathrm{BCR}-\mathrm{ABL}$
LTHSCs (500 cells per mouse) at 4 weeks after transplantation revealed increased numbers of donor LTHSCs in mice receiving MPL hi LTHSCs compared with those receiving MPL ${ }^{\text {lo }}$ LTHSCs (Supplemental Figure 2D) and confirmed that LTHSCs regenerated from MPL ${ }^{\text {hi }}$ LTHSCs demonstrated significantly higher MPL 
A

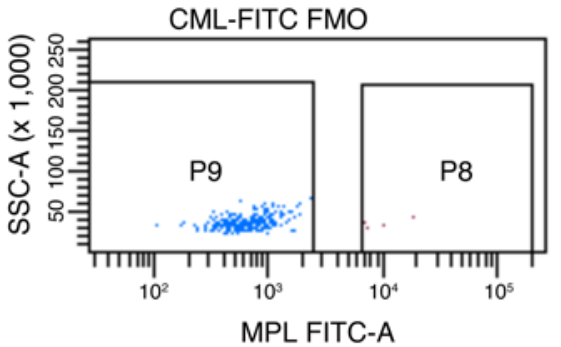

C

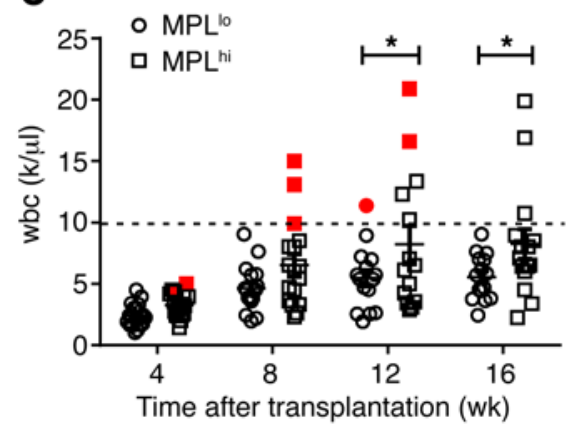

$\mathbf{F}$

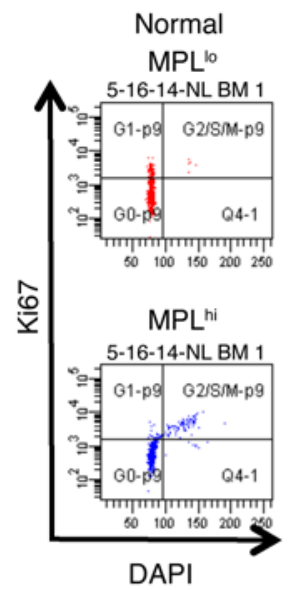

H $\circ G_{0}$

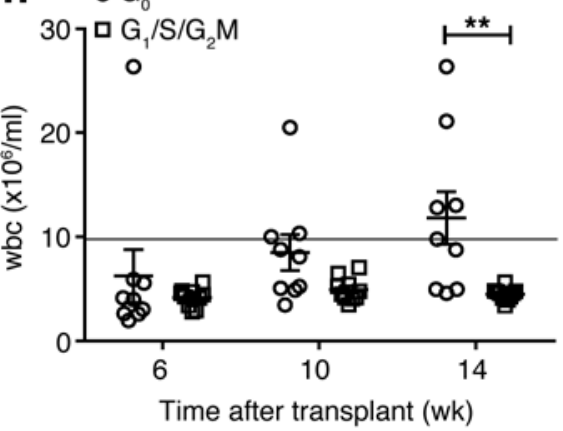

D

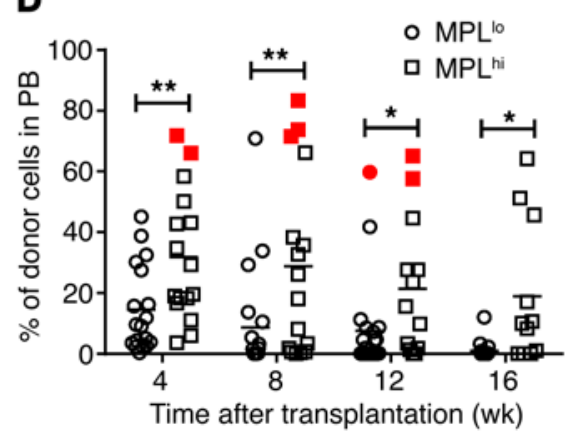

G $\quad \mathrm{CML}$

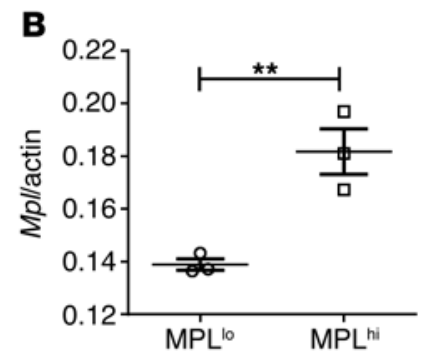

E

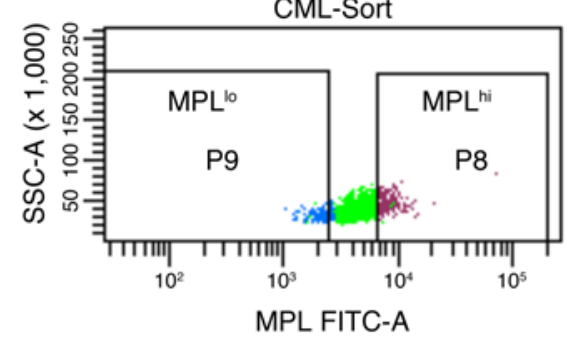

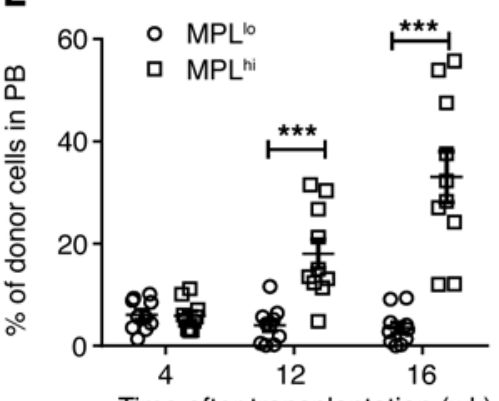

Time after transplantation (wk)

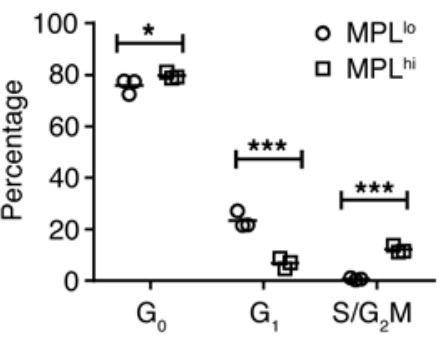

$\mathrm{MPL}^{1 \circ}$
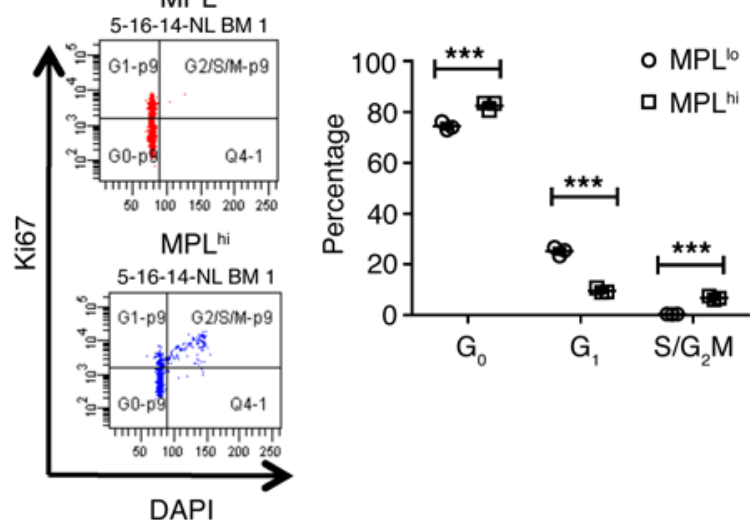

○ MPL'10

$\mathrm{MPL}^{\mathrm{ni}}$

I $\circ G_{0}$

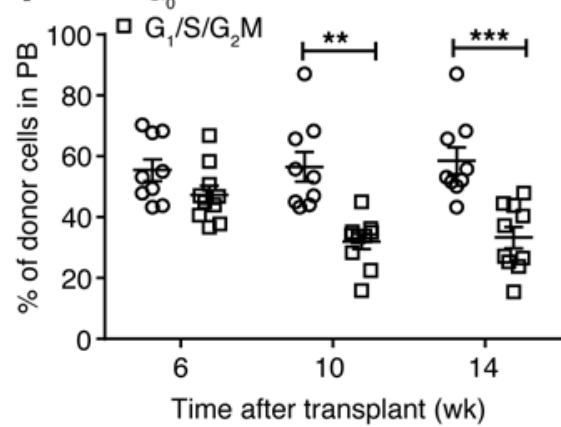

J $\circ G_{0}$

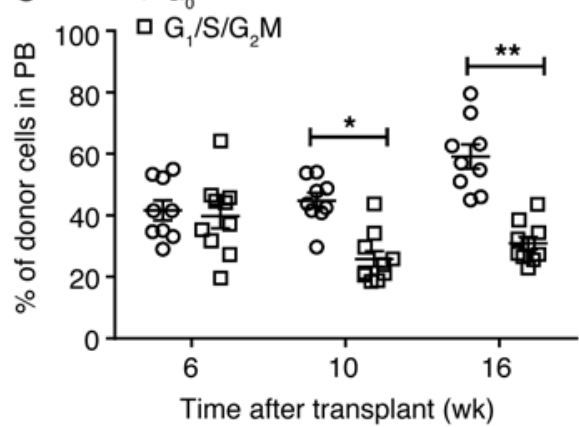

Figure 3. Enhanced leukemogenic capacity of MPL ${ }^{\text {hi }}$ LTHSCs. (A) Representative flow cytometry plots of LTHSCs with high (MPL ${ }^{\text {hi }}$ ) and low (MPL ${ }^{10}$ ) levels of cell surface MPL expression. (B) Mpl mRNA expression in MPL hi and MPL ${ }^{10}$ LTHSCs cells. (C) wbc count and (D) engraftment of CML CD45.1 cells in the PB of CD45.2 recipients after transplantation with CML MPL hi and MPL ${ }^{10}$ LTHSCs (200 cells per mouse). Red symbols represent the mice that were sick and euthanized or found dead. The dashed lines represent the upper limit of the normal wbc count, distinguishing leukemic from nonleukemic mice. (E) Engraftment of CD45.1 normal cells in the PB of CD45.2 recipients after transplantation with normal MPL ${ }^{\text {hi }}$ and MPL ${ }^{10}$ LTHSCs (50 cells per mouse). Flow cytometry plot and summary graph showing the cell cycle status of $\mathrm{MPL}^{\mathrm{hi}}$ and $\mathrm{MPL}{ }^{10} \mathrm{LTHSCs}$ for $(\mathbf{F})$ normal and $(\mathbf{G}) \mathrm{CML}$ mice determined by Ki-67 and DAPI

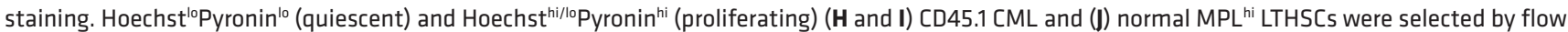
cytometry and transplanted into CD45.2 recipients (100 cells per mouse, 10 mice per group). Engraftment in PB was monitored as shown every 4 weeks for 16 weeks or until mice developed $\mathrm{CML}$ or had to be euthanized. Results represent mean \pm SEM. ${ }^{*} P<0.05$; ${ }^{*} P<0.01$; ${ }^{* * *} P<0.001$. 2-way ANOVA was used to assess significance. 
A

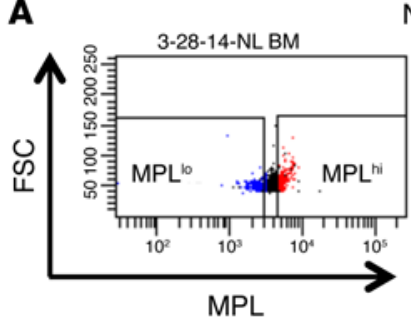

Normal

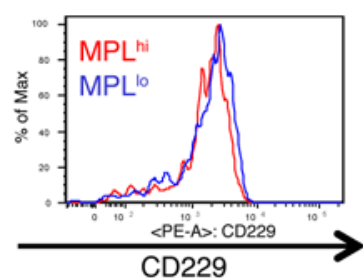

CML
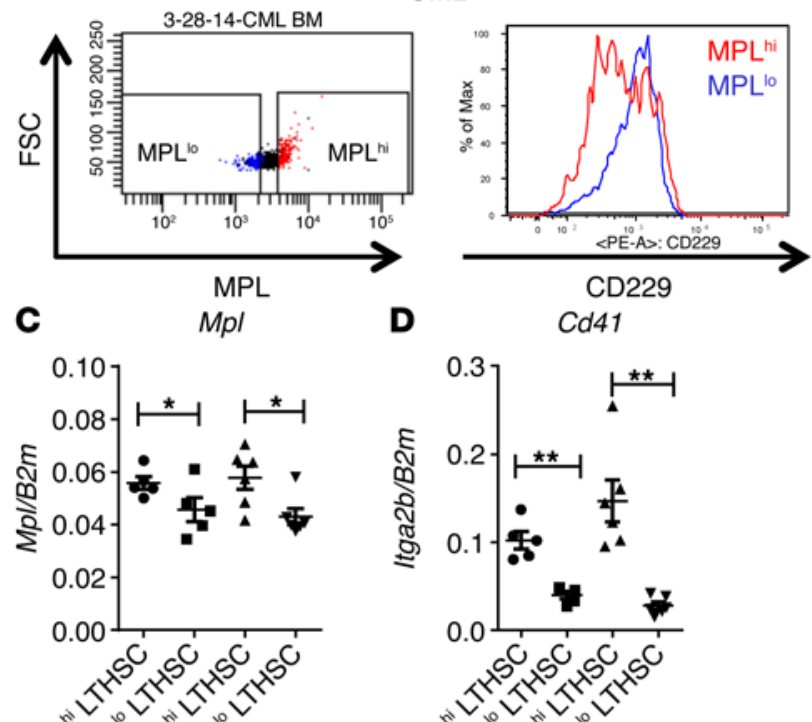

$35.35 .35,35$

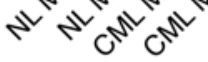

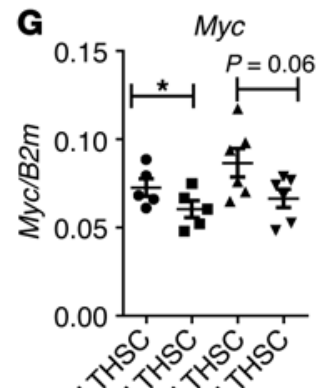

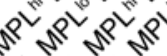

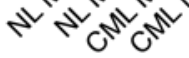
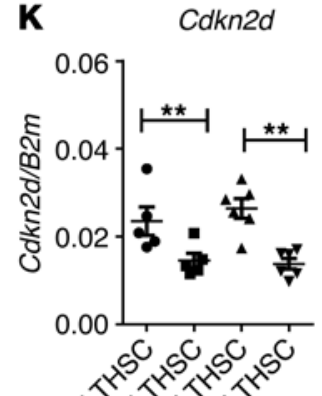

$3.5 n$

$3.4 c^{2} a^{2}$

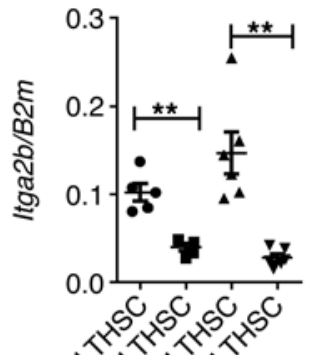

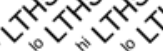

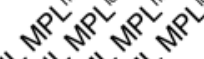

$4.420,0$
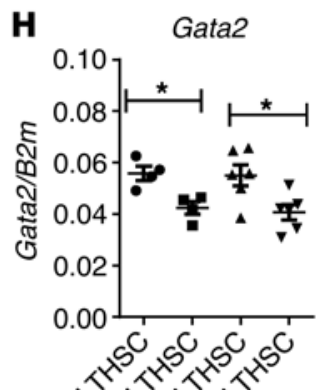

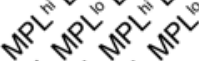

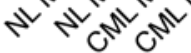

L

Cdkn1b

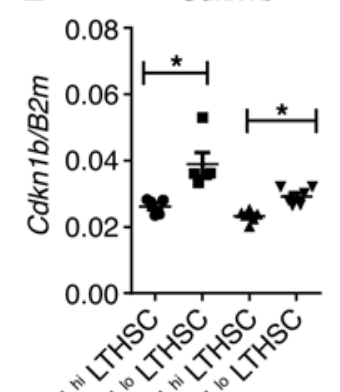

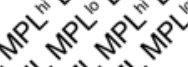

$3,5 a^{2} a_{2}$

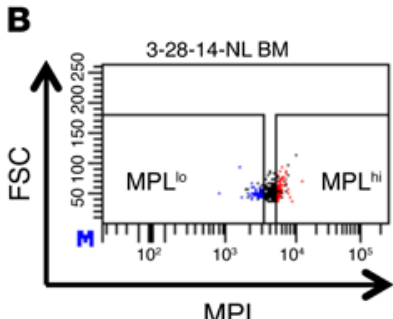

Normal

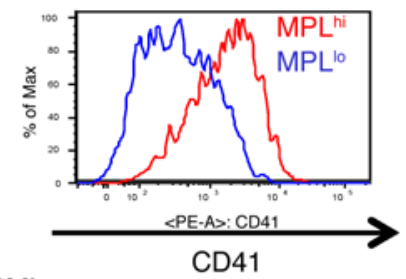

CML
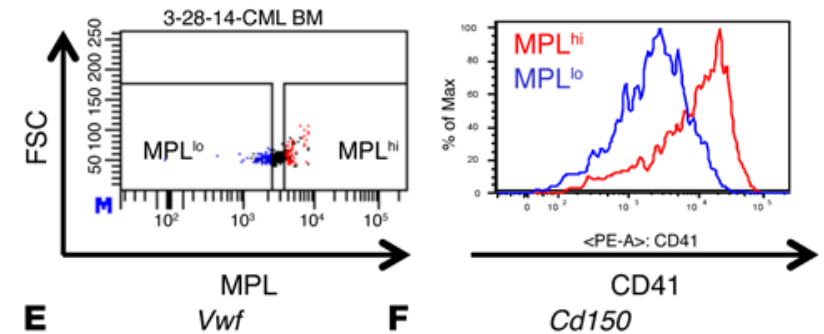

F $\quad$ Cd150

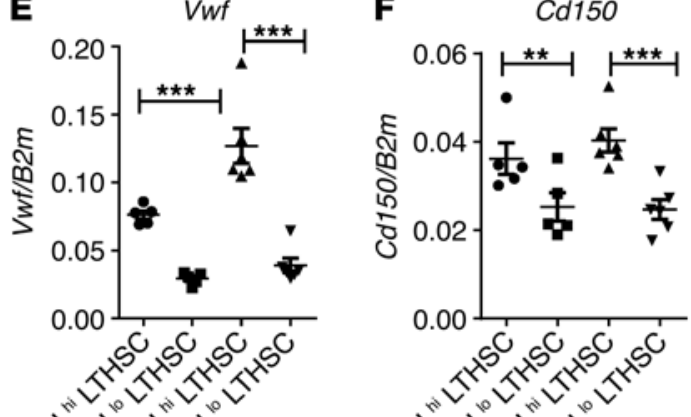

3.535

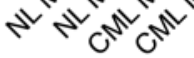

I

$B C l-X L$

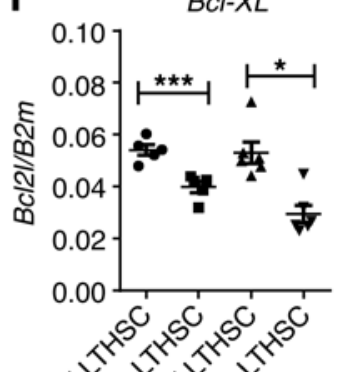

$5.50^{2} a^{2}$

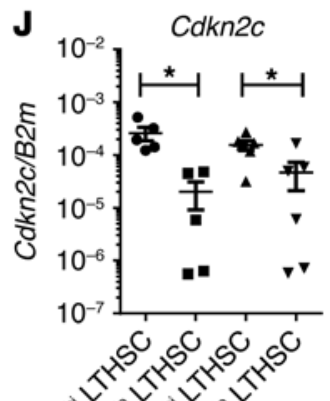

$3.55 .35,5 s$

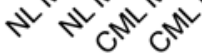

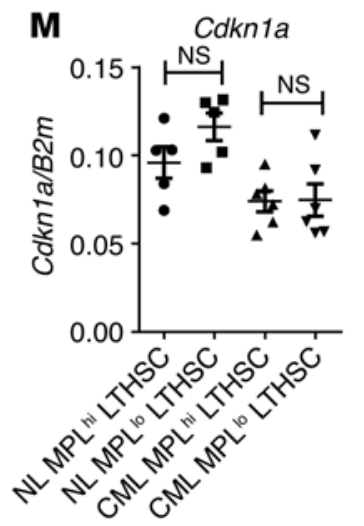

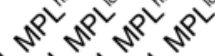

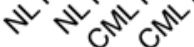
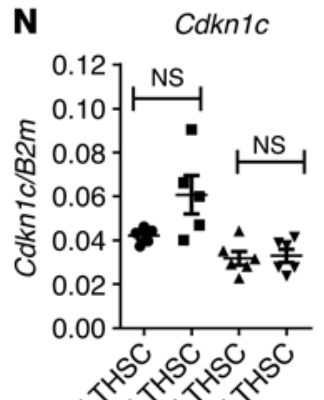

s.s.s.3.

$4.42 a^{2} a^{2}$

Figure 4. Expression of megakaryocytic lineage, stem cell, and cell cycle regulatory genes in MPL hi LTHSCs. Flow cytometry plots showing coexpression of (A) CD229 and (B) CD41 on normal and CML MPL hi and MPL ${ }^{10}$ LTHSCs cells. mRNA expression of (C-E) megakaryocytic lineage, (F-I) stem cell, and $(\mathbf{J}-\mathbf{N})$ cell cycle regulatory genes in normal and $C M L ~ M P L$ hi and $M P L^{10}$ LTHSCs analyzed by Q-RT-PCR. Results represent mean \pm SEM. ${ }^{*} P<0.05$; ${ }^{* *} P<0.01 ;{ }^{* *} P<0.001$. Student's $t$ test was used to assess significance. 
expression compared with those generated from MPL ${ }^{\text {lo }}$ LTHSCs (Supplemental Figure 2E), indicating maintenance of higher MPL expression after transplantation.

The cell cycle status of MPL ${ }^{\text {hi/lo }}$ LTHSCs was evaluated by Ki-67 and DAPI labeling. A significant increase in the proportion of $G_{0}$ phase cells was seen in both normal (Figure 3F) and CML (Figure 3G) MPL hi LTHSCs compared with that in MPL ${ }^{\text {lo }}$ LTHSCs. On the other hand, a significant increase in cells in $\mathrm{S} / \mathrm{G}_{2} / \mathrm{M}$ phase was seen in MPL ${ }^{\text {hi }}$ LTHSCs compared with that in MPL ${ }^{\text {lo }}$ LTHSCs. This increase in LTHSCs in $\mathrm{S} / \mathrm{G}_{2} / \mathrm{M}$ phase in MPL ${ }^{\text {hi }}$ LTHSCs compared with that in MPL ${ }^{\text {lo }}$ LTHSCs was confirmed by EdU labeling (Supplemental Figure 2, F and G). These observations suggest that MPL ${ }^{\text {hi }}$ LTHSCs contain both an increased proportion of quiescent cells and a population of actively cycling cells. To determine the functional stem cell capacity of quiescent versus cycling MPL ${ }^{\text {hi }}$ LTHSCs, quiescent (Hoechst ${ }^{\text {lo }}$ Pyronin $^{\text {lo }}, G_{0}$ ) and cycling (Hoechst ${ }^{\text {hi/lo }}$ Pyronin ${ }^{\text {hi }}, G_{1} / S / G_{2} / M$ ) MPL ${ }^{\text {hi }}$ LTHSCs from normal and CML mice (CD45.1) were selected by flow cytometry (Supplemental Figure 2H) and were transplanted into CD45.2 recipients (100 cells per mouse). Quiescent CML MPL ${ }^{\text {hi }}$ LTHSCs showed significantly enhanced long-term engraftment and leukemogenic capacity compared with cycling LTHSCs (Figure 3, H and I). Normal MPL hi LTHSCs also demonstrated increased longterm engraftment compared with cycling LTHSCs (Figure 3J).

$M P L^{h i}$ LTHSCs show enhanced expression of megakaryocytic lineage genes and a distinct pattern of HSC and cell cycle gene expression. The lack of expression of CD229 is a marker of LTHSCs that are more quiescent and have high myeloid regenerative potential (18). BCR-ABL ${ }^{+}$MPL hi LTHSCs showed lower CD229 expression compared with MPL ${ }^{\text {lo }}$ LTHSCs, suggesting that they may represent a more primitive LTHSC subpopulation (Figure 4A and Supplemental Figure 3A). However, CD229 expression was similar in normal MPL hi and MPL ${ }^{\text {lo }}$ LTHSCs. Since MPL is the receptor for THPO, the primary extrinsic regulator for platelet differentiation, we also evaluated expression of two other platelet-lineage-related genes, $C d 41$ and $V w f$, in MPL ${ }^{\text {hi }}$ LTHSCs. LTHSCs expressing these genes demonstrate enhanced platelet reconstitution, long-term myeloid lineage bias, and self-renewal $(19,20)$. We observed higher cell surface expression of CD41 on MPL hi LTHSCs compared with that on MPL lo LTHSCs from both BCR-ABL and control mice (Figure $4 \mathrm{~B}$ and Supplemental Figure 3B). We also observed increased mRNA expression of $\mathrm{Mpl}$ (Figure 4C), Cd41 (Figure 4D), and Vwf (Figure 4E) by Q-RT-PCR analysis in MPL hi LTHSCs compared with MPL ${ }^{\text {lo }}$ LTHSCs from normal and BCR/ABL mice. MPL ${ }^{\text {hi }}$ LTHSCs also demonstrated increased expression of the HSC-associated genes, Cd15O, Gata2, Bcl-XL, and $M y c$, compared with MPL lo LTHSCs (Figure 4, F-I). In general, gene expression differences between $\mathrm{MPL}^{\text {hi }}$ and MPL ${ }^{\text {lo }}$ cells followed similar patterns for BCR-ABL and control LTHSCs. We observed increased expression of cell cycle inhibitory genes, Cdkn2c (also known as p18) and Cdkn2d (also known as p19), in MPL ${ }^{\text {hi }}$ LTHSCs compared with MPL ${ }^{\text {lo }}$ LTHSCs (Figure 4, J and K), whereas expression of $C d k n 1 b$ (also known as p27), which regulates progenitor cells but not stem cell proliferation and pool size, was reduced in the MPL ${ }^{\text {hi }}$ LTHSCs (Figure $4 \mathrm{~L}$ and ref. 21). Levels of Cdkn1a and Cdkn1c were not significantly different between $\mathrm{MPL}^{\text {hi }}$ and MPL ${ }^{\text {lo }}$ LTHSCs (Figure 4, M and N).
Knockdown of MPL expression diminishes growth and leukemogenic capacity of CML LTHSCs. We used lentivirus vectors to express anti-MPL or control shRNA and GFP in BCR-ABL and normal LTHSCs. Q-RT-PCR and flow cytometry confirmed effective knockdown of $M p l$ mRNA and protein expression (Figure 5, A-C). CML LTHSCs expressing MPL shRNA demonstrated significantly reduced in vitro cell growth and CFC potential in the presence of THPO (Supplemental Figure 4, A and B), and an increase in apoptotic cells (Supplemental Figure 4C), compared with control shRNA-expressing cells. MPL knockdown also resulted in a significant increase in $G_{0} / G_{1}$ cells and a reduction in S phase cells (Supplemental Figure 4, D and E). To test in vivo engraftment, MPL shRNA- and control shRNA-expressing LTHSCs from BCR-ABL mice (CD45.1) were transplanted into congenic CD 45.2 recipients. Recipients of MPL knockdown BCRABL LTHSCs showed reduced wbc counts and $\mathrm{GFP}^{+}$donor cells in peripheral blood (PB) compared with controls (Figure 5, D and E). Whereas ten of seventeen mice receiving control shRNAexpressing LTHSCs developed leukemia within 16 weeks, only one of fifteen mice receiving MPL shRNA-expressing LTHSCs developed leukemia (Figure 5, D and E, and Supplemental Figure 4F). MPL shRNA-expressing LTHSCs also generated significantly reduced engraftment of $\mathrm{GFP}^{+}$donor cells in the $\mathrm{BM}$ and spleen at 16 weeks compared with control shRNA-expressing LTHSCs (Supplemental Figure 4G). Transplanting normal LTHSCs expressing MPL shRNA into congenic recipients also resulted in markedly reduced donor cell engraftment in $\mathrm{PB}$ compared with controls (Figure 5F) and reduced donor cell engraftment in BM and spleen at 16 weeks (Supplemental Figure 4H). MPL shRNAexpressing LTHSCs engrafted in mice were confirmed to have reduced $M p l$ mRNA expression in vivo (Figure 5G). MPL knockdown significantly reduced the frequency of LTHSCs in $G_{0}$ as well as in $S / G_{2} / M$ in vivo (Figure $5 H$ ). On the other hand, no significant difference in apoptosis was seen in vivo (Figure 5I). These observations indicate a complex role for MPL in regulating CML LTHSC quiescence as well as transition through cell cycle in vivo.

We analyzed the effect of MPL knockdown on p-STAT3 and p-STAT5 expression in BCR-ABL LTHSCs. Expression of p-STAT3 and p-STAT5 peaked at 30 minutes following exposure to THPO (10 ng/ml) (Figure 6, A and B). Knockdown of MPL expression resulted in reduced THPO-stimulated p-STAT3 and p-STAT5 expression in BCR-ABL LTHSCs (Figure 6, A and B). MPL shRNAexpressing LTHSCs were analyzed for expression of genes associated with THPO signaling using multiplex Q-RT-PCR. We observed significantly reduced expression of STAT target genes, including Bcl-XL, Myc, Pim1, Osm, and Hif2a, in LTHSCs with MPL knockdown compared with that in control LTHSCs from both BCR-ABL and wild-type mice (Figure 6, C-G) (22). Expression of other genes associated with MPL signaling, including Hoxa9, Hoxb4, and Tie2, was also reduced in MPL shRNAexpressing LTHSCs from normal, but not BCR-ABL, mice (Figure $\left.6, \mathrm{H}^{-} \mathrm{J}\right)$. Expression of the megakaryocytic lineage genes $V w f$ and $C d 41$ was not significantly affected by MPL knockdown (Figure $6, \mathrm{~K}$ and $\mathrm{L}$ ). In addition, we observed reduced expression of cell cycle regulatory genes, Cdkn1b, Cdkn1c (also known as p57), and Cdkn2d, following MPL knockdown in normal LTHSCs, consistent with previous reports of reduced levels of these genes 
A

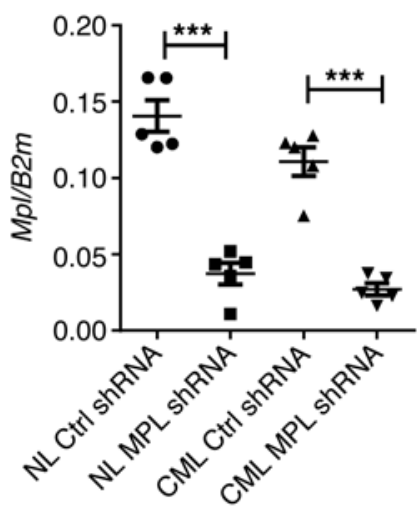

D
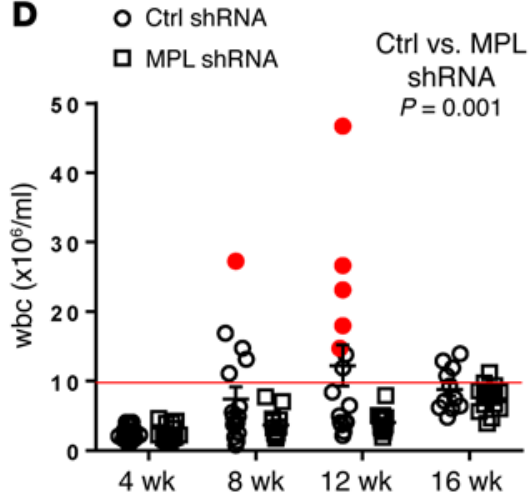

G
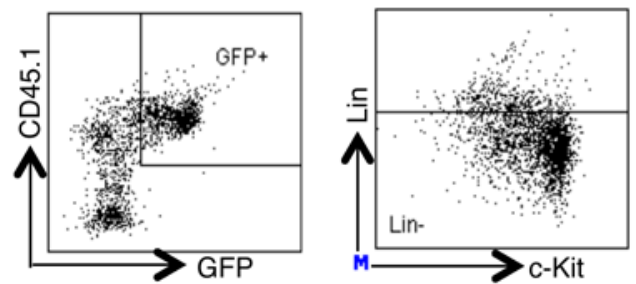

B
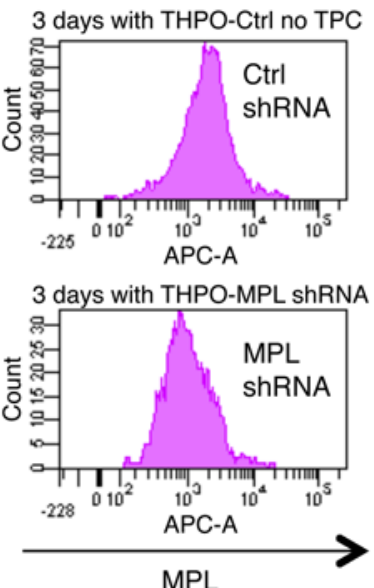

E $\quad$ O Ctrl ShRNA
C

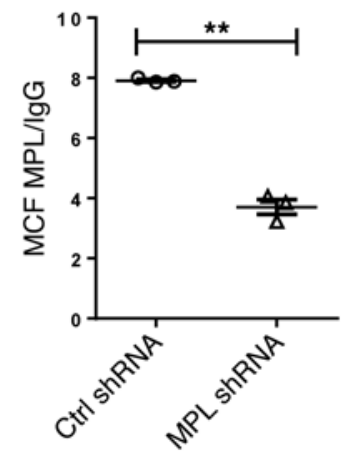

$\mathbf{F}$

Ctrl vs. MPL shRNA

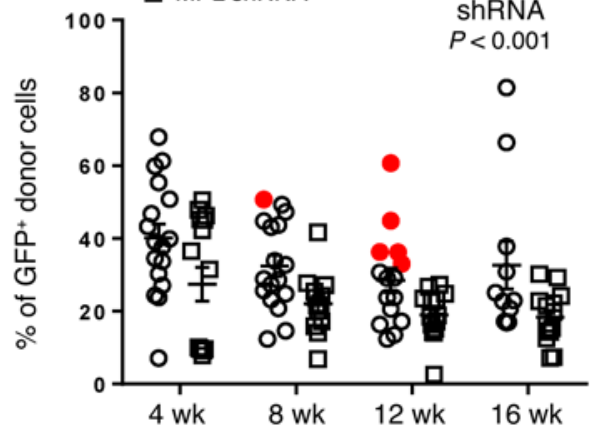

- Ctrl shRNA

口 MPL ShRNA

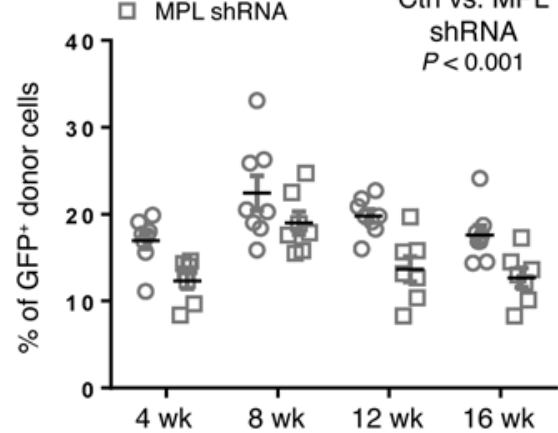

H

H Ctrl shRNA
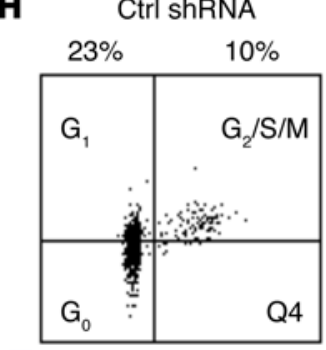

M $66 \%$
MPL ShRNA

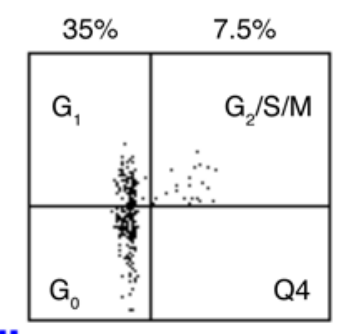

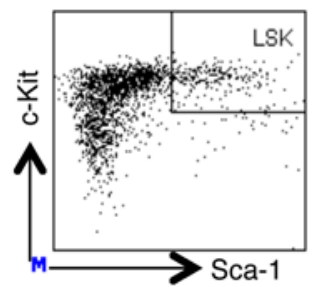

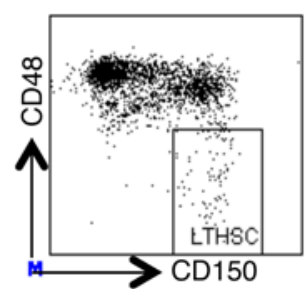

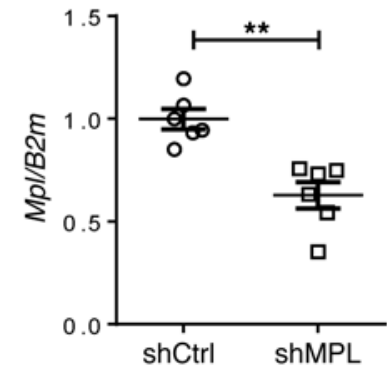

I

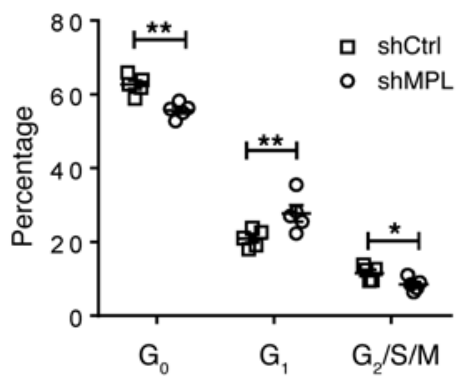

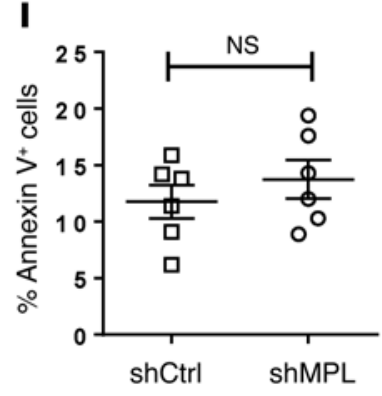

Figure 5. RNAi-mediated knockdown of MPL expression reduces the leukemogenic capacity of CML LTHSCs. (A) Mpl mRNA and (B) protein expression in normal and CML LTHSCs transduced with lentivirus vectors expressing MPL shRNA or control shRNA and GFP. CML LTHSCs were transduced with lentivirus vectors expressing MPL or control shRNA and cultured with and without THPO for 72 hours. (C) MPL protein expression. CD45.1 normal and CML LTHSCs transduced with MPL shRNA or control shRNA lentivirus were transplanted into CD45.2 recipients. (D) wbc count and (E) engraftment of GFP+ CML donor cells and (F) normal donor cells in the PB of recipients. Red symbols represent the mice that were sick and euthanized or found dead. BM cells were obtained from mice receiving GFP+ LTHSCs expressing MPL shRNA (shMPL) or control shRNA (shCtrl) at 6 weeks after transplantation. (C) Mpl gene expression in GFP+ LTHSCs. (H) Cell cycle and (I) apoptosis of engrafted GFP+ LTHSCs expressing MPL shRNA or control shRNA was analyzed. Results represent mean \pm SEM. ${ }^{*} P<0.05 ;{ }^{* *} P<0.01 ;{ }^{* *} P<0.001$. Student's $t$ test was used to assess significance. 
A

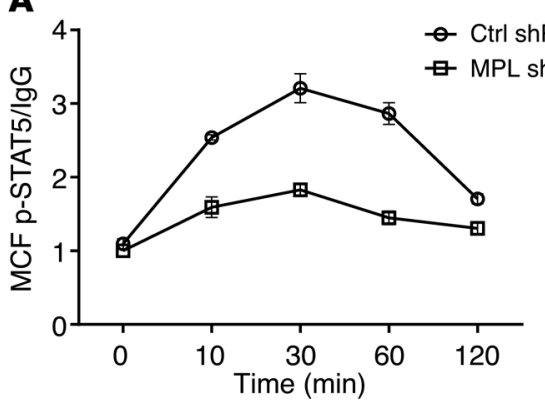

B

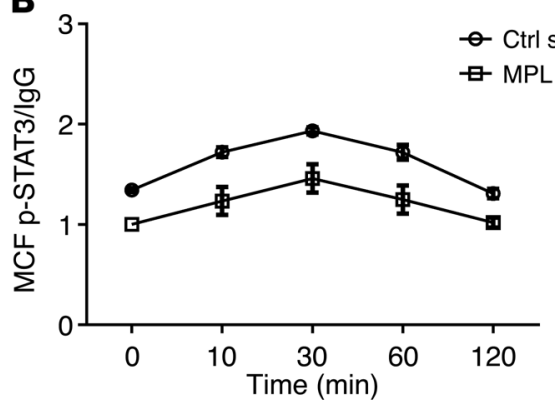

C

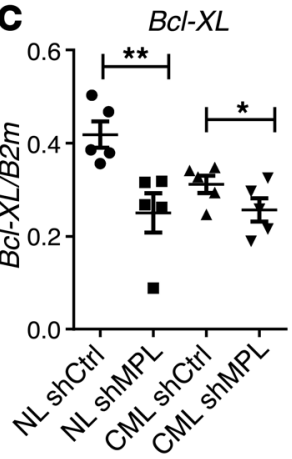

D
E

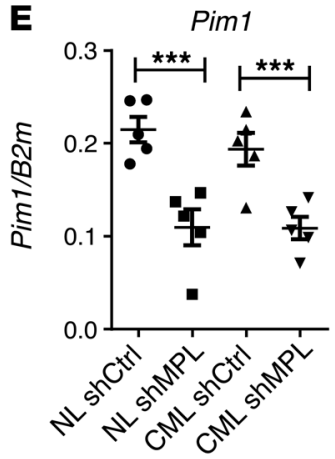

$\mathbf{F}$
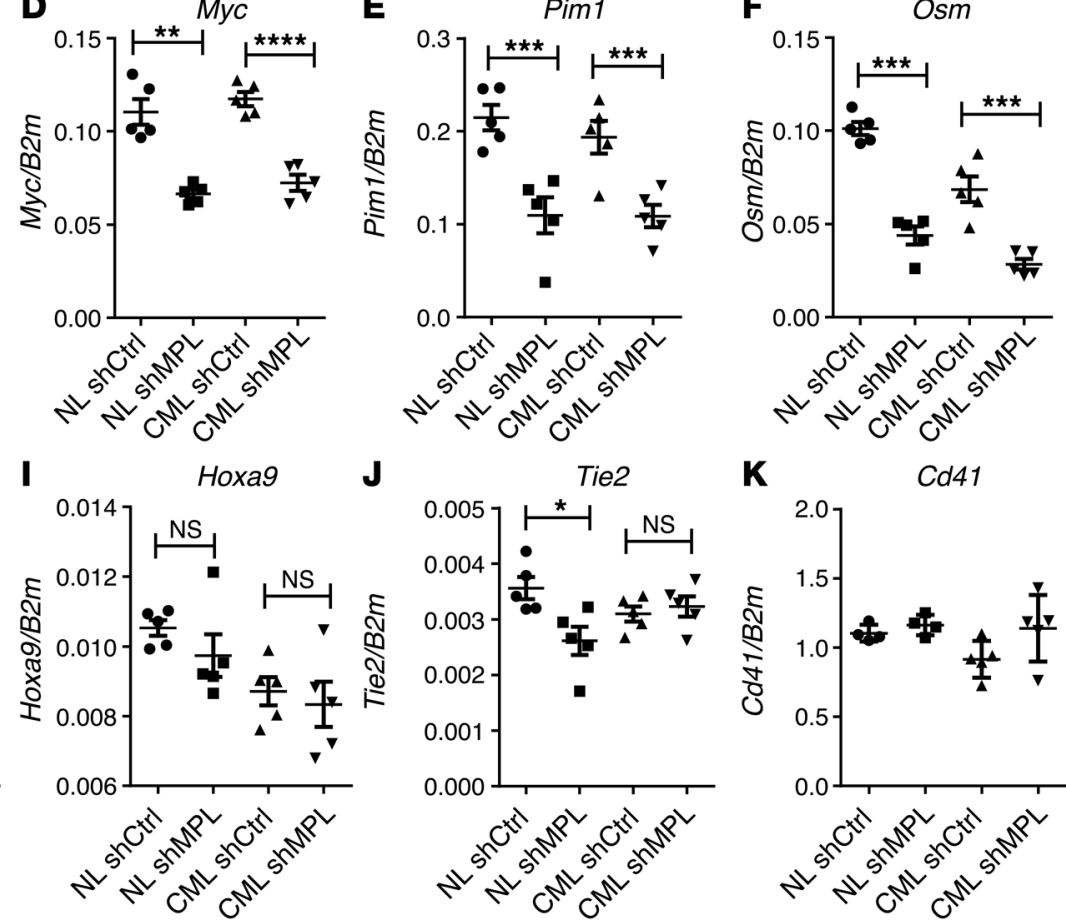

$\mathbf{N}$

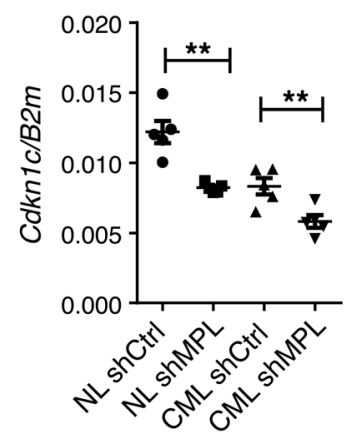

Tie2

J

K

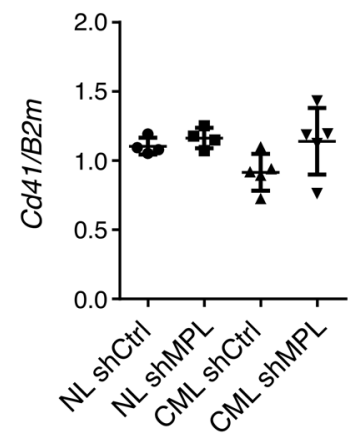

$\mathbf{0}$
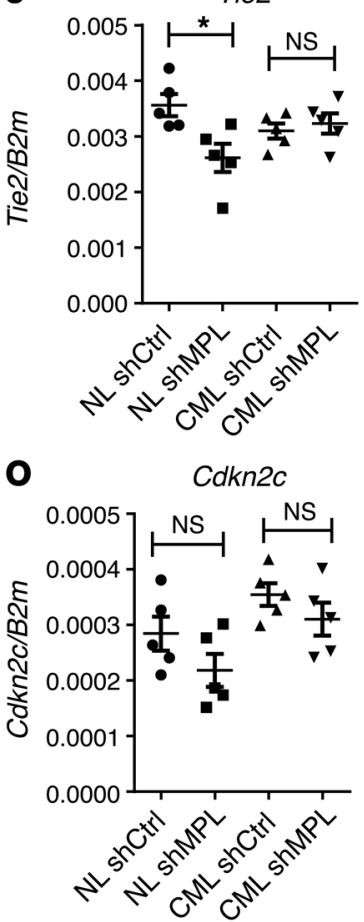

$\mathbf{P}$

Cdkn2d

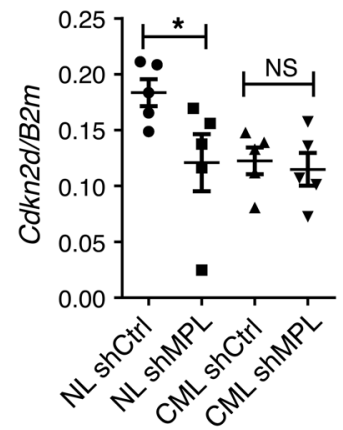

G

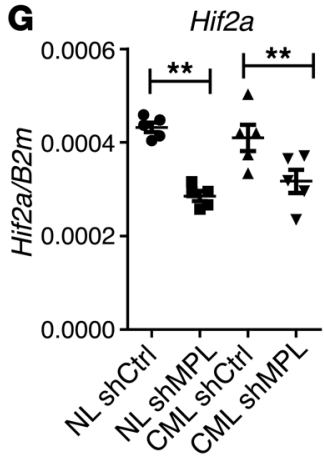

$\mathbf{L}$

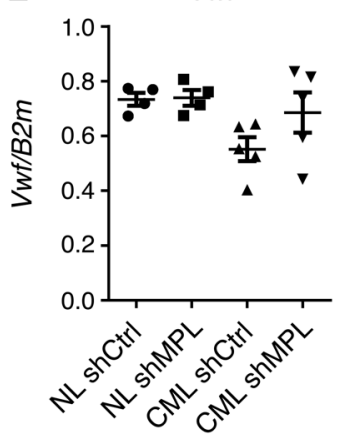

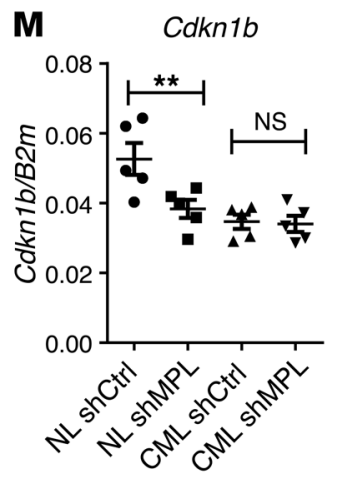

Figure 6. Effect of MPL knockdown on STAT signaling and STAT target gene and cell cycle regulatory gene expression. Expression of (A) p-STAT5 and (B) p-STAT3 in CML LTHSCs transduced with MPL shRNA or control shRNA lentivirus analyzed by flow cytometry. Expression of mRNA for (C-G) STAT target genes, (H-J) MPL-related genes, (K and $\mathbf{L}$ ) megakaryocytic lineage genes, and (M-P) cell cycle regulatory genes in normal and CML LTHSCs transduced with MPL shRNA or control shRNA lentivirus analyzed by Q-RT-PCR. Results represent mean \pm SEM. ${ }^{*} P<0.05 ;{ }^{* *} P<0.01 ;{ }^{* * *} P<0.001 ;{ }^{* * *} P<0.0001$. Student's $t$ test was used to assess significance.

in Thpo-deleted mice (Figure 6, M-P, and ref. 14). On the other hand, MPL knockdown in BCR-ABL LTHSCs resulted in reduced expression of $C d k n 1 c$ but not other CDK inhibitors.

Human MPL ${ }^{\text {hi }}$ LTHSCs also showed increased in vitro and in vivo cell growth. We evaluated the relationship of MPL expression and the proliferative potential of LTHSCs from patients with CML and from normal cord blood samples. As seen with murine cells, $\mathrm{CD}_{4}{ }^{+} \mathrm{CD} 38^{-} \mathrm{CD}^{-} 0^{+}$LTHSCs showed the highest MPL expression among $\mathrm{CD}_{3} 4^{+}$progenitor cells (Supplemental Figure 5A). MPL expression was reduced in $\mathrm{CML} \mathrm{CD}^{+} 4^{+}$cells compared with that in normal CD34 ${ }^{+}$cells (Supplemental Figure $5 \mathrm{~B}$ ) but did not significantly differ between CML and normal LTHSCs (Figure 7A). 

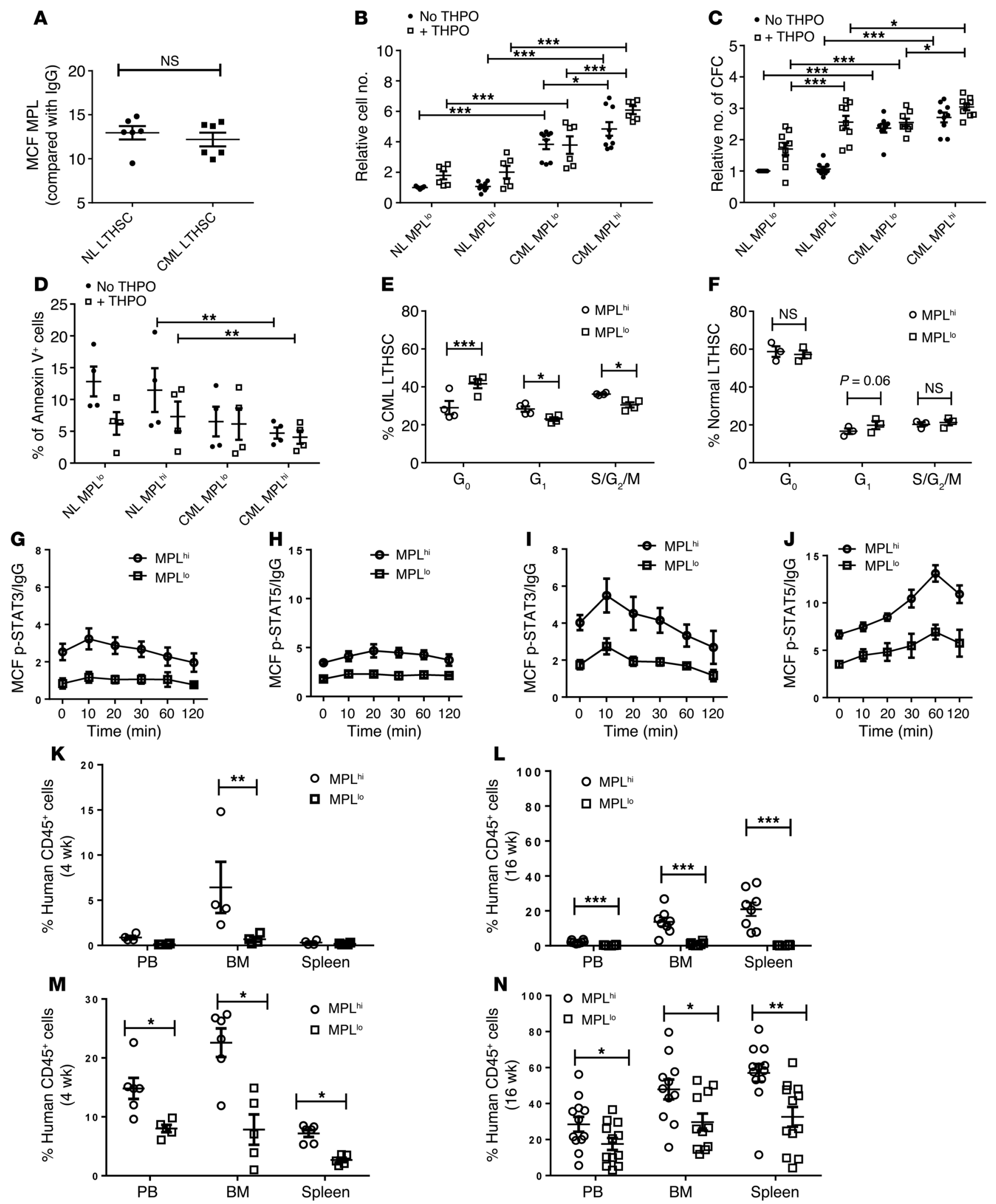
Figure 7. Human CML MPL hi LTHSCs show enhanced proliferative and regenerative capacity. (A) MPL expression in human normal and CML CD34 + CD38-CD90 + LTHSCs analyzed by flow cytometry. (B) Cell growth, (C) CFC, (D) apoptosis of normal and CML MPL ${ }^{\text {hi }}$ and MPL ${ }^{10}$ LTHSCs cultured in SFEM medium for 72 hours with or without THPO $(10 \mathrm{ng} / \mathrm{ml})$. (E and F) Cell cycling of CML and normal $\mathrm{MPL}^{\text {hi }}$ and $M P L^{10}$ LTHSCs cultured in SFEM medium for 72 hours in the presence of THPO $(10 \mathrm{ng} / \mathrm{ml})$. Combined results of $\mathrm{p}$-STAT3 and p-STAT5 expression by flow cytometry in ( $\mathbf{G}$ and $\mathbf{H}$ ) normal and (I and J) CML MPL hi and MPL ${ }^{10}$ LTHSCs in the presence of THPO $(10 \mathrm{ng} / \mathrm{ml})(n=4)$. Normal and CML MPL ${ }^{\text {hi }}$ and MPL ${ }^{10}$ LTHSCs were sorted and transplanted into NSC mice. Engraftment of human CD45+ cells in PB, $B M$, and spleens at 4 weeks and 16 weeks from ( $\mathbf{M}$ and $\mathbf{N}$ ) normal and ( $\mathbf{K}$ and L) CML LTHSCs. Results represent mean \pm SEM of results from 4 to 13 patients with $\mathrm{CML}$. ${ }^{*} P<0.05$; ${ }^{*} P<0.01 ;{ }^{* *} P<0.001$. 2-way ANOVA was used to assess significance.

Human CML MPL hi LTHSCs cultured in the presence or absence of THPO showed significantly increased cell growth and CFC frequency compared with CML MPL ${ }^{10}$ LTHSCs and significantly increased cell growth and CFC generation compared with normal MPL hi LTHSCs (Figure 7, B and C). CML MPL hi LTHSCs also showed significantly reduced apoptosis with and without THPO compared with their normal counterparts (Figure 7D). The cell cycle status of MPL ${ }^{\text {hi/lo }}$ LTHSCs with and without THPO $(10 \mathrm{ng} / \mathrm{ml})$ was evaluated by Ki-67 and DAPI labeling. A significant decrease in the proportion of $G_{0}$ phase cells and increase in the proportion of $G_{1}$ and $S / G_{2} / M$ phase cells was seen in CML MPL ${ }^{\text {hi }}$ LTHSCs compared with that in MPL ${ }^{10}$ LTHSCs in the presence of THPO (Figure 7E). A significant difference in the proportion of cells in different phases was not seen in normal MPL ${ }^{\text {hi }}$ LTHSCs compared with MPL ${ }^{\text {lo }}$ LTHSCs in the presence of THPO (Figure 7F). No significant difference was seen in cell cycling between $\mathrm{MPL}^{\mathrm{hi}}$ and MPL ${ }^{\text {lo }}$ LTHSCs cultured without THPO from both normal patients and patients with CML (Supplemental Figure 5F). Human CML MPL ${ }^{\text {hi/o }}$ LTHSCs were analyzed for STAT3 and STAT5 activity. Both baseline and THPO-stimulated p-STAT3 and p-STAT5 levels were markedly higher in CML MPL ${ }^{\text {hi }}$ LTHSCs compared with those in MPL ${ }^{\text {lo }}$ LTHSCs and in CML compared with normal MPL ${ }^{\text {hi }}$ LTHSCs (Figure 7, G-J). Interestingly, the p-STAT5 response peaked at 60 minutes in CML LTHSCs, as compared with a peak at 20 minutes in normal LTHSCs, indicating alterations in kinetics as well as amplitude of p-STAT3/5 response to THPO signaling in CML LTHSCs compared with normal LTHSCs. We next evaluated engraftment of $\mathrm{CML}_{\mathrm{MPL}} \mathrm{L}^{\text {hi }}$ and $\mathrm{MPL}^{\mathrm{lo}} \mathrm{CD} 34^{+} \mathrm{CD} 38^{-} \mathrm{CD} 90^{+}$ cells in immunodeficient NSG mice. It is a well-recognized limitation of human CML xenograft models that engraftment is relatively low and that transplanted mice do not develop leukemia or die of disease, possibly reflecting the lack of specific human factors in the murine BM microenvironment. Despite these limitations, xenograft models remain the best measure of the longterm engraftment capacity of human CML LTHSCs. Following transplantation into NSG mice, CML MPL ${ }^{\text {hi }}$ LTHSCs generated a significantly higher engraftment of human $\mathrm{CD} 45^{+}$cells in $\mathrm{BM}$ at both 4 and 16 weeks compared with MPL ${ }^{\text {lo }}$ LTHSCs (Figure 7, K and L). CML MPL hi LTHSCs generated significantly higher levels of $\mathrm{CD}_{3} 4^{+}$progenitors and $\mathrm{CD} 33^{+}$myeloid cells and lower levels of $\mathrm{CD} 19^{+}$lymphoid cells in the $\mathrm{BM}$ of recipient mice at 16 weeks compared with MPL ${ }^{10}$ LTHSCs (Supplemental Figure 5C). However, BCR-ABL expression was similar in CML MPL ${ }^{\text {hi }}$ and $\mathrm{MPL}^{\text {lo }}$
LTHSC-derived human cells engrafted in BM of NSG mice (Supplemental Figure 5D). These results indicate that engrafting cells are $\mathrm{BCR}-\mathrm{ABL}^{+}$but do not rule out partial engraftment of normal cells. These results indicate that MPL expression also identifies human CML LTHSCs with enhanced regenerative potential, similar to what was observed for murine CML LTHSCs. We also observed higher engraftment of normal MPL ${ }^{\text {hi }}$ LTHSCs compared with MPL ${ }^{10}$ LTHSCs in NSG mice at 4 and 16 weeks (Figure 7, M and $\mathrm{N}$, and Supplemental Figure 5E).

Human MPL ${ }^{\text {hi }}$ LTHSCs show reduced sensitivity to nilotinib. We evaluated the effect of treatment with the TKI nilotinib (NIL) on selected human MPL ${ }^{\text {hi }}$ and MPL ${ }^{\text {lo }}$ LTHSCs. Although NIL treatment inhibited growth (Figure 8A) and CFC capacity (Figure $8 \mathrm{~B}$ ) of both $\mathrm{MPL}^{\text {hi }}$ and MPL ${ }^{\text {lo }}$ CML LTHSCs, we saw significantly greater inhibition of MPL ${ }^{10}$ LTHSCs compared with that of MPL hi LTHSCs. NIL treatment enhanced apoptosis of MPL $^{\text {lo }}$ LTHSCs but not MPL ${ }^{\text {hi }}$ LTHSCs compared with controls and induced significantly greater apoptosis of NIL-treated $M^{M P L^{\text {lo }}}$ LTHSCs compared with MPL ${ }^{\text {hi }}$ LTHSCs (Figure 8C). On the other hand, NIL treatment inhibited cell cycling of both $\mathrm{MPL}^{\mathrm{hi}}$ and MPL ${ }^{\mathrm{lo}} \mathrm{CML}$ LTHSCs (Figure 8D). These results suggest that MPL hi LTHSCs demonstrate lower levels of sensitivity to TKI compared with MPL ${ }^{\text {lo }}$ LTHSCs.

Role of downstream signaling pathways in mediating MPL effects in CML LTHSCs. Since JAK/STAT and MEK signaling can be activated downstream of MPL stimulation by THPO, we evaluated the role of JAK/STAT and MEK signaling in mediating MPL effects in CML LTHSCs. MPL ${ }^{\text {hi }}$ and MPL $^{\text {lo }}$ human CML LTHSCs were cultured with THPO in the presence of AZD1480 (a JAK inhibitor) and PD0325901 (a MEK inhibitor). Evaluation of p-ERK, p-STAT3, and p-STAT5 by phospho-flow analysis confirmed inhibition of JAK/STAT and MEK signaling in AZD1480- and PD0325901-treated cells, respectively (Figure 8E). Treatment with either AZD1480 or PD0325901 resulted in significantly reduced cell growth (Figure $8 \mathrm{~F}$ ) and $\mathrm{CFC}$ capacity (Figure $8 \mathrm{G}$ ) of MPL ${ }^{\text {hi }}$ and MPL ${ }^{\text {lo }}$ LTHSCs, with significantly greater inhibition seen after AZD1480 treatment compared with that after PD0325901 treatment. Treatment with AZD1480 resulted in significantly increased apoptosis of MPL hi but not MPL ${ }^{\text {lo }}$ LTHSCs (Figure 8H) and inhibited cell cycling of both MPL $^{\text {hi }}$ and MPL ${ }^{\text {lo }}$ LTHSCs (Figure 8I), whereas treatment with PD0325901 did not significantly increase apoptosis in either MPL $^{\text {hi }}$ or MPL ${ }^{\text {lo }}$ LTHSCs (Figure $8 \mathrm{H}$ ), modestly inhibited cycling of MPL hi LTHSCs, and did not significantly inhibit cycling of MPL $^{\text {lo }}$ LTHSCs (Figure 8I). These results indicate a role for both JAK/STAT and MAPK signaling in MPL-mediated regulation of cell cycling in CML LTHSCs but suggest a larger role for JAK/ STAT signaling in MPL-mediated antiapoptotic effects and enhanced cell growth.

\section{Discussion}

There is an increasing appreciation that HSCs demonstrate heterogeneity in proliferative, self-renewal, and differentiation properties at a clonal level, reflecting a complex interaction of intrinsic and extrinsic factors (6). On the other hand, heterogeneity of function of oncogene-transformed stem cells in leukemia has not been well studied. In this study, we evaluated gene expression 


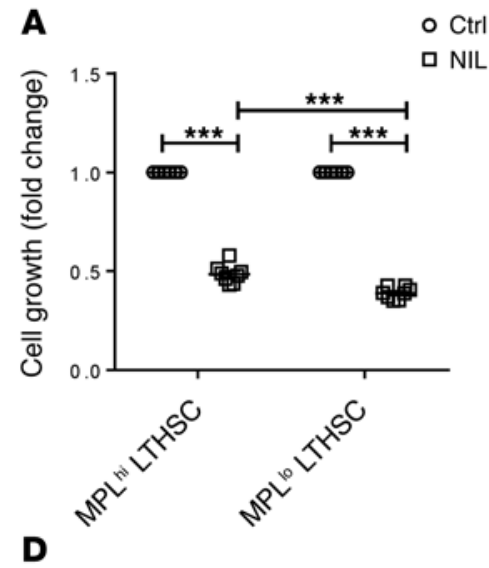

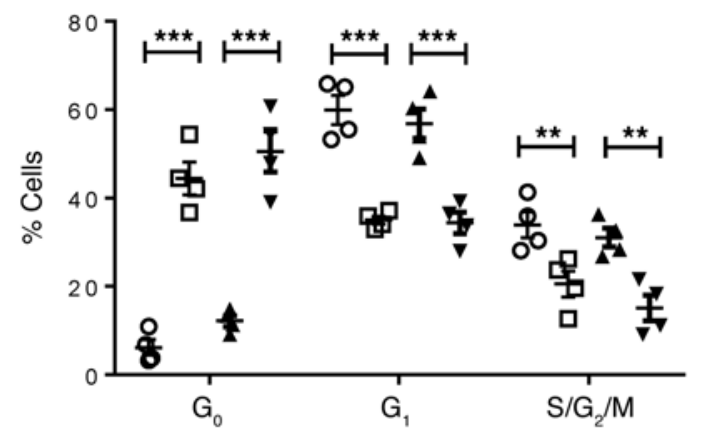

$\mathbf{F}$

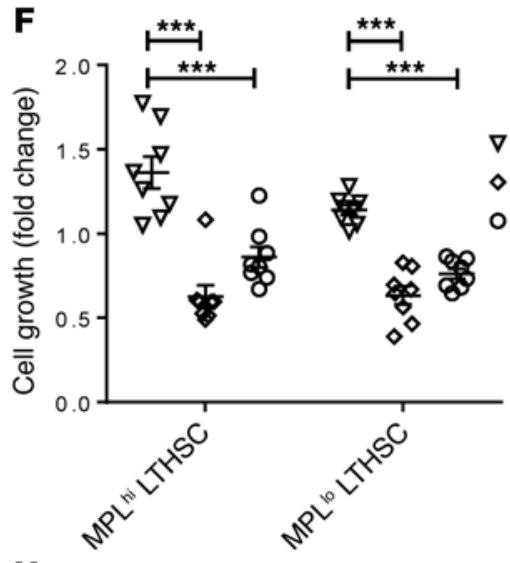

H

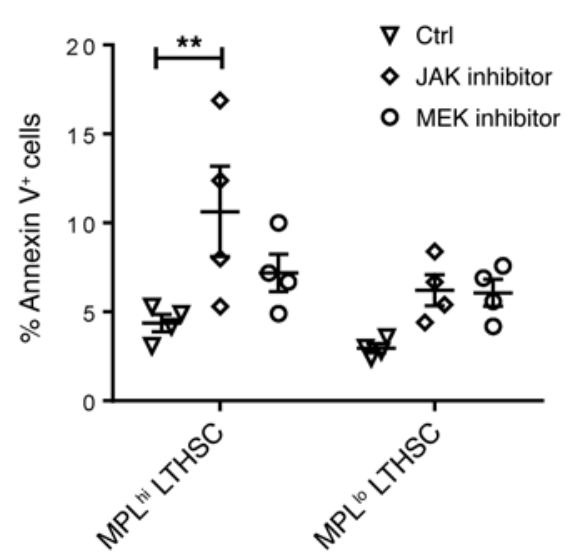

- JAK inhibitor

- MEK inhibitor

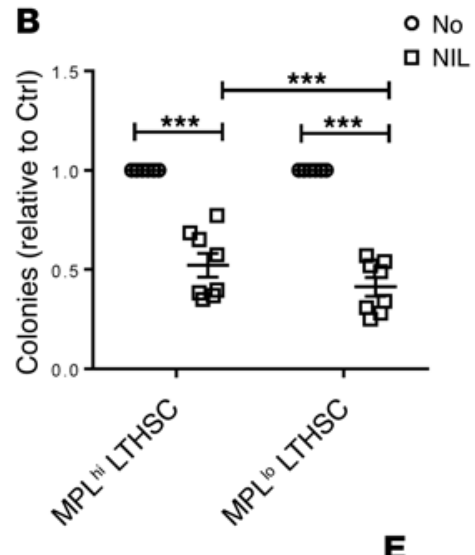

C
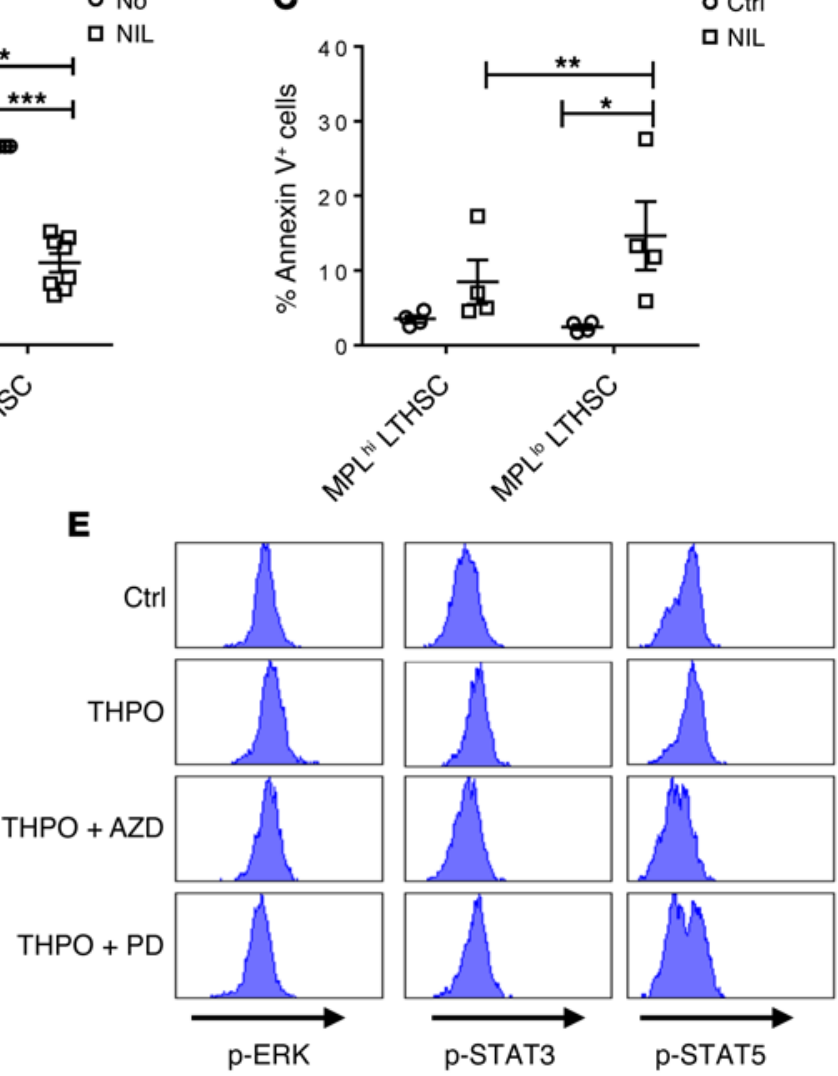

○ MPLi $\mathrm{Ctrl}$

$\square$ MPL ${ }^{\text {hi }}$ NIL

1 $M P L^{\circ} \mathrm{Ctrl}$

$\checkmark \mathrm{MPL}^{10} \mathrm{NIL}$
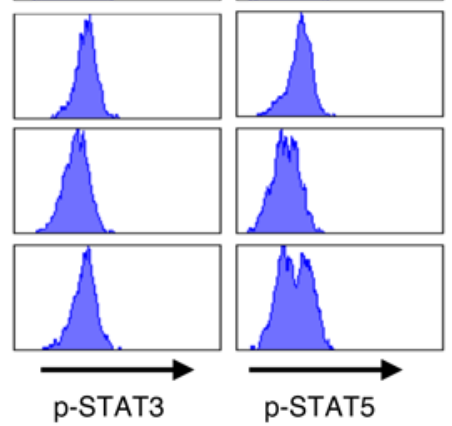
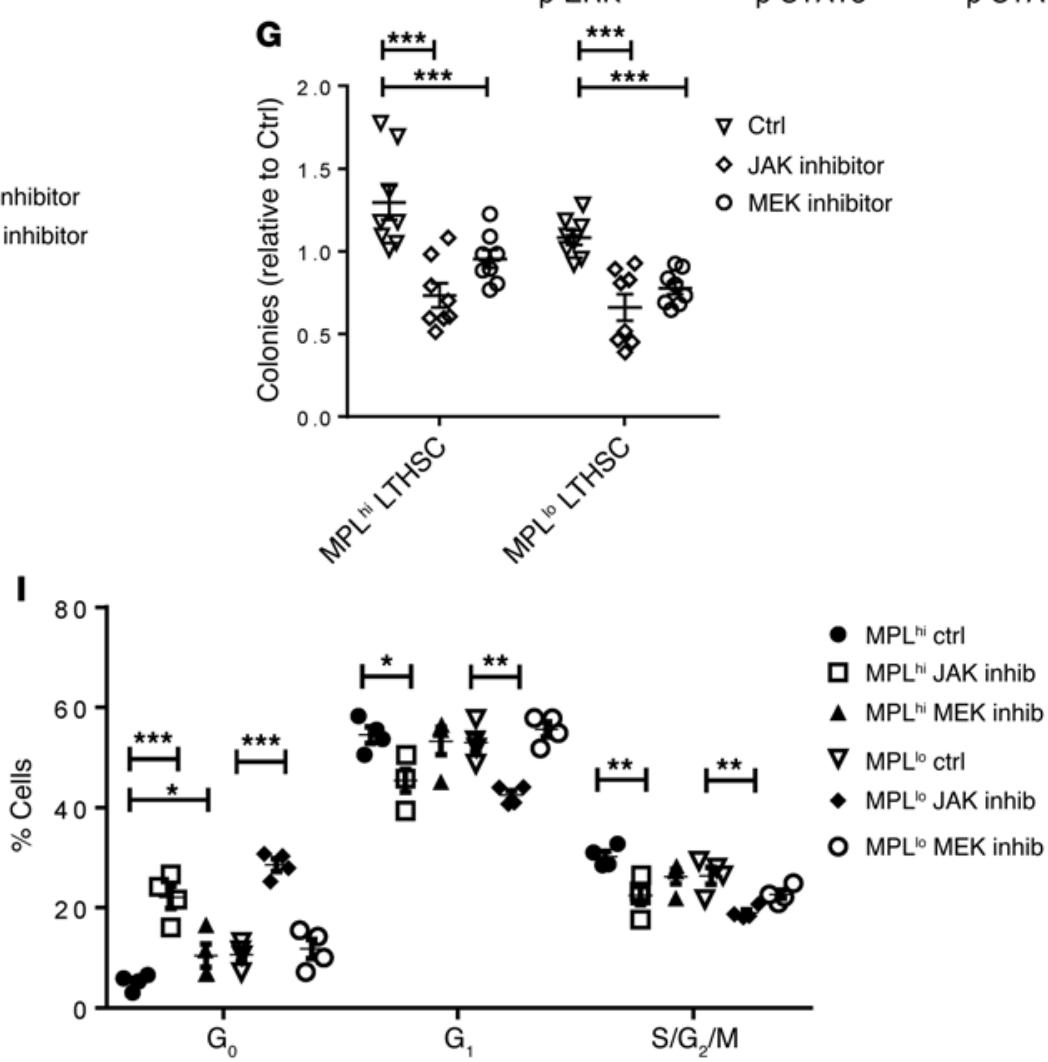
Figure 8. Sensitivity of human MPL ${ }^{\text {hi }}$ LTHSCs to BCR-ABL, JAK, and MEK inhibitors. Human CML MPL hi and MPL ${ }^{10}$ LTHSCs (Lin ${ }^{-C D} 34^{+}$CD38-CD90+) were sorted by flow cytometry and treated with NIL for 3 days. Then, (A) cell growth, (B) CFC, (C) apoptosis, and (D) cell cycle were analyzed. (E) CML $M P L^{\text {hi }}$ and $M P L^{10} L T H S C s$ were cultured with and without THPO in the presence of the JAK inhibitor AZD1480 or the MEK inhibitor PD0325901, and p-ERK, p-STAT3, and p-STAT5 expression was checked by flow cytometry. CML MPL ${ }^{\text {hi }}$ and $M P L^{10}{ }^{10}$ THSCs were cultured with JAK or MEK inhibitor for 3 days and (F) cell growth, (G) CFC capacity, (H) apoptosis, and (I) cell cycle were checked. ${ }^{*} P<0.05$; ${ }^{* *} P<0.01$; ${ }^{* *} P<0.001$. 2-way ANOVA was used to assess significance.

patterns associated with heterogeneity in leukemogenic capacity of BCR-ABL-expressing LTHSCs. Evaluation of differential expression of a preselected group of genes, of known importance in stem cell function, in mediating LTHSC heterogeneity led to the identification of increased expression of the THPO receptor MPL in murine $\mathrm{BCR}-\mathrm{ABL}^{+}$LTHSCs with leukemogenic potential compared with those without leukemogenic potential. Murine and human BCR-ABL-expressing LTHSCs selected for higher MPL expression showed enhanced in vitro expansion and increased leukemia-initiating capacity in vivo compared with those with lower MPL expression. Finally, human CML LTHSCs with higher MPL expression demonstrated reduced sensitivity to the BCR-ABL TKI NIL but increased sensitivity to a JAK inhibitor. These results identify MPL expression as a marker as well as a key regulator of leukemogenic potential and drug sensitivity of CML LTHSCs. It is recognized that additional genes beyond those studied here could contribute to the functional heterogeneity of LSCs in CML. Indeed, RNA-Seq analysis identified that gene expression patterns associated with the immune response were downregulated in BCR-ABL ${ }^{+}$LTHSCs from mice that developed leukemia compared with nonleukemic mice, suggesting a potential role for the immune response in regulating the leukemogenic potential of BCR-ABL ${ }^{+}$LTHSCs. This observation opens up further avenues for future investigation.

Increased MPL expression was also associated with enhanced proliferation and engraftment capacity of normal murine and human LTHSCs. These results are consistent with previous reports that stem cell activity segregated with MPL expression in murine fetal stem cell-enriched populations (16). Murine CML and normal MPL hi LTHSCs demonstrate increased expression of megakaryocytic lineage genes and a distinct pattern of HSC and cell cycle gene expression. This gene expression pattern suggests similarities to a recently described molecularly and functionally distinct subset of "platelet-primed" LTHSCs, which express vWF and other platelet-specific genes, and appear to be at the apex of the HSC hierarchy (19). Platelet-primed HSCs are characterized by dependence on THPO for maintenance, platelet-regenerating capacity, long-term myeloid lineage bias, enhanced self-renewal capacity, and capability of generating lymphoid-biased HSCs. Similarly, LTHSCs expressing the platelet integrin CD41 also exhibit megakaryocyte gene priming, show marked myeloid bias, and possess long-term repopulation and serial transplantation capacity (20). Therefore, the enhanced leukemogenic capacity of MPL ${ }^{\text {hi }}$ LTHSCs could be related to their being derived from platelet-primed LTHSCs with increased self-renewal capacity and myeloid bias.
CML MPL hi LTHSCs also demonstrate low expression of CD229, a LTHSC phenotype associated with increased quiescence and greater myeloid regenerative potential (18).

Murine CML and normal MPL hi LTHSCs demonstrate increased proportions of quiescent $G_{0}$ cells associated with increased expression of $C d k n 2 c$ and $C d k n 2 d$. However, an increase in S phase cells was also observed, suggesting that MPL hi LTHSC population also contains an actively cycling subset of cells. Inhibition of MPLexpression inhibited leukemogenicity ofCMLLTHSCs, suggesting that, besides identifying LTHSCs with enhanced leukemogenic potential, enhanced MPL expression directly contributes to CML LTHSC self-renewal and regenerative capacity. MPL inhibition also reduced engraftment of normal LTHSCs, consistent with previous studies, showing that THPO signaling through MPL regulates maintenance as well as after transplant expansion of HSCs $(13,23,24)$. MPL-deficient mice have reduced HSC frequency and competitive repopulating capacity (15). Normal HSCs transplanted into $\mathrm{Thpo}^{-/}$recipient mice show markedly reduced expansion (14). Our results suggest that, in addition to its known role in normal HSCs, THPO/MPL signaling also plays an important role in maintenance and expansion of CML LSCs. The effects of THPO/MPL signaling on cell cycle are complex. In vivo THPO administration leads initially to an increase in quiescence and upregulation of CDK inhibitor expression, followed by increased cycling. In contrast, THPO exposure in vitro increases cycling of quiescent HSCs (25). Our results indicate that THPO/ MPL signaling has similarly complex effects on CML LTHSC cell cycling. MPL knockdown in murine BCR-ABL $\mathrm{BLTHSC}^{+}$reduced cell cycling in vitro and in vivo but also reduced LTHSC quiescence in vivo. These results suggest a dual role for MPL signaling both in maintaining a quiescent LSC population and in promoting LSC cycling and leukemic cell expansion. Importantly, longterm engraftment and leukemia-initiating capacity were enriched within the quiescent subpopulation of MPL hi LTHSCs.

Both murine and human CML LTHSCs show enhanced THPO-stimulated STAT3/5 activation. Knockdown of MPL expression in murine CML and normal LTHSCs resulted in reduced THPO-stimulated STAT3 $/ 5$ activation, with reduced expression of STAT target genes Bcl-XL, Myc, Piml, Osm, and Hif2a. MPL knockdown did not alter expression of the megakaryocytic lineage genes $C d 41$ and $V w f$, suggesting that the expression of platelet-related genes is not dependent on MPL signaling. Although the effects of MPL knockdown on downstream genes were similar in CML and normal LTHSCs, several differences were also noted. For example, knockdown of MPL expression resulted in reduced expression of Hoxa9 and Hoxb4 in normal LTHSCs, consistent with previous reports of reduced expression of several Hox genes in HSCs from Thpo- mice. On the other hand, Hox gene expression was not altered in CML LTHSCs, suggesting that they may not play a critical role in MPL regulation of LSC function. MPL knockdown reduced Cdkn1b, Cdkn1c, and $C d k n 2 d$ in normal LTHSCs, consistent with previous studies showing increased HSC cycling and decreased expression of CDK inhibitors after exposure to neutralizing anti-MPL antibodies and in $T h p^{-/-}$mice. In contrast, MPL knockdown reduced only Cdkn1c in CML LTHSCs, indicating that MPL interacts differently with the cell cycle regulatory machinery in leukemic 
LTHSCs compared with normal LTHSCs. Interestingly, among CDK inhibitors, CDKN1C has been reported to play a predominant role in quiescence and maintenance of adult HSCs (26).

In CML cells, BCR-ABL can directly phosphorylate and activate STAT5 without a requirement for JAKs (27). However, JAK2 appears to be important for STAT activation downstream of cytokine signaling in CML cells (28). Increased baseline and THPO-stimulated p-STAT3 and p-STAT5 expression in CML MPL $^{\text {hi }}$ LTHSCs compared with that in normal MPL ${ }^{\text {hi }}$ LTHSCs suggests that BCR-ABL can potentiate MPL signaling through the JAK/STAT signaling pathway. Since receptor internalization plays a necessary role in MPL signaling, enhanced MPL signaling may explain in part reduced receptor expression seen in murine CML LTHSCs compared with that in normal LTHSCs (29). Altered MPL signaling is also a feature of BCR-ABL-negative myeloproliferative neoplasms, in which, in addition to more commonly seen JAK2 V617F mutations, MPL mutations leading to constitutive activation of JAK2, STAT3, and STAT5 signaling are often observed (30). In AML LSC, high STAT signaling activity is associated with poor prognosis $(31,32)$.

Resistance of CML LSCs to elimination by TKI treatment is an important area of investigation in CML. LSC persistence and consequent risk of relapse leads to life-long dependence on TKI treatment to maintain remission in the bulk of patients with CML. We found that human CML MPL ${ }^{\text {hi }}$ LTHSCs demonstrated reduced TKI-mediated inhibition of growth and resisted TKImediated inhibition of survival compared with MPL ${ }^{\text {lo }}$ LTHSCs. TKI resistance may be related in part to THPO-induced JAK/ STAT activation in CML cells. Induction of LSC quiescence by THPO signaling may further contribute to resistance of MPL hi LTHSCs to TKI. In addition, signaling through the TPHO/MPL/ BCL-XL pathway has been shown to contribute to survival and self-renewal in human RUNX1-ETO-induced leukemia $(33,34)$. Finally, THPO may also potentiate resistance to antileukemia treatment by enhancing DNA repair, which could limit cytotoxicity of DNA-damaging treatments (35). Our findings support further investigation of approaches to antagonize MPL signaling as a potential therapeutic strategy to eliminate leukemia-initiating LTHSCs. Such approaches could include specific neutralizing antibodies to MPL and inhibitors that also enhance receptor internalization and degradation or inhibition of downstream mechanisms, such as JAK/STAT signaling (36). We have recently reported that JAK2 inhibition with ruxolitinib can enhance elimination of chronic phase CML CD34 ${ }^{+}$cells in combination with BCR-ABL TKI (37). The present study shows that JAK2 and MEK inhibition reduced human CML MPL hi LTHSC cell cycling and cell growth. JAK2 inhibition also enhanced apoptosis of MPL ${ }^{\text {hi }}$ LTHSCs. Since MPL ${ }^{\text {hi }}$ LTHSCs demonstrate reduced sensitivity to BCR-ABL TKI alone, these observations provide a strong rationale for further exploration of the use of additional JAK/STAT inhibition to target CML LSC resistant to BCR-ABL TKI alone.

Our results may also be relevant to the observation that residual $\mathrm{BCR}-\mathrm{ABL}^{+}$hematopoietic cells can be detected in patients with $\mathrm{CML}$ maintaining treatment-free remissions, without disease recurrence, following hematopoietic cell transplantation, interferon- $\alpha$, and TKI treatment $(4,38,39)$. The long-term persistence of leukemic cells indicates the presence of BCR-ABL ${ }^{+}$LTHSCs, whereas lack of leu- kemia development indicates that such LTHSCs have impaired leukemogenic capacity, possibly related to reduced self-renewal and proliferation $(40,41)$. This implies that leukemogenic LTHSCs are critical targets for therapeutics, whereas minimal residual disease resulting from persistence of nonleukemogenic LTHSCs may not confer risk of relapse. Our results may also have relevance for explaining the detection of $B C R-A B L$ transcripts in blood cells from healthy individuals without CML (42). The cell context in which $B C R-A B L$ gene mutation occurs is likely to be critical, and the mutation may need to occur in LTHSCs with high self-renewing potential in order to induce leukemia. THPO receptor agonist drugs are effective in the treatment of refractory immune thrombocytopenia and are being investigated for treatment of BM failure syndromes $(43,44)$. However, clonal evolution or progression to AML has been recognized as a concern when using THPO receptor agonists in patients with aplastic anemia and MDS, and registry data suggests a possible association between these agents and AML in patients with immune thrombocytopenia (45). Our findings that TPO signaling can sustain or expand CML LTHSCs indicate the need for caution in using these agents in patients with or at risk for leukemia, with careful evaluation of their risks and benefits.

\section{Methods}

Mice. Inducible transgenic SCL-tTA/BCR-ABL mice in the CD45.1 $\mathrm{FVB} / \mathrm{N}$ background were maintained with administration of tetracycline in the drinking water (0.5 g/l) (10). For some experiments, SCLtTA/BCR-ABL mice were crossed with transgenic GFP-expressing mice (FVB.Cg-Tg [ACTB-EGFP] B5Nagy/J, The Jackson Laboratory) to facilitate tracking of donor $\mathrm{GFP}^{+}$cells in recipient $\mathrm{FVB} / \mathrm{N}$ mice after transplantation. Alternatively, recipient mice in the CD45.2 FVBN background (a gift from Emmanuelle Passegue, UCSF, San Francisco, California, USA) were used to allow tracking of donor CD45.1 cells after transplant. Recipient mice were 6 to 8 weeks old and were irradiated at $900 \mathrm{cGy}$. If not indicated, BCR-ABL expression was induced for 3 weeks by tetracycline withdrawal.

Samples. Cord blood samples were provided by StemCyte. CML samples were obtained from patients in chronic phase who had not received treatment with TKI. Mononuclear cells were isolated using Ficoll separation. CD $34^{+}$cells were isolated using a positive magnetic bead selection protocol (Miltenyi).

Antibodies. Details for all antibodies used are provided in Supplemental Table 4.

Selection of LTHSC subsets. Murine cells obtained from PB, BM (both tibias and femurs), and spleens were analyzed using a LSRII flow cytometer (BD). Mouse (LSK Flt3 ${ }^{-} \mathrm{CD}^{-} 15 \mathrm{O}^{+} \mathrm{CD} 48^{-}$cells) and human ( $\mathrm{Lin}^{-}$ $\mathrm{CD} 4^{+} \mathrm{CD} 38^{-} \mathrm{CD}^{-} 0^{+}$cells) LTHSCs were isolated by flow cytometry sorting using a FACSAria flow cytometer (BD). To isolate LTHSCs based on MPL expression, cells were also labeled with an anti-mouse MPL antibody (generated in-house), Children's Hospital of Philadelphia, Philadelphia, Pennsylvania, USA) or an anti-human MPL antibody (CD110, 562199, BD), and LTHSC fractions (20\%) with the highest $\left(\mathrm{MPL}^{\mathrm{hi}}\right)$ and lowest $\left(\mathrm{MPL}^{\mathrm{lo}}\right)$ expression were selected by flow cytometry.

In vitro proliferation, apoptosis, and flow cytometry assays. Human MPL $^{\text {hi }}$ and MPL ${ }^{\text {lo }}$ LTHSCs were cultured in Stemspan serum-free medium (SFEM, StemCell Technologies) supplemented with low concentrations of growth factors (granulocyte-macrophage colony-stimulating factor, $200 \mathrm{pg} / \mathrm{ml}$; granulocyte colony-stimulating factor, $1 \mathrm{ng} / \mathrm{ml}$; stem cell 
factor [SCF], $200 \mathrm{pg} / \mathrm{ml}$; leukemia inhibitory factor, $50 \mathrm{pg} / \mathrm{ml}$; macrophage inflammatory protein- $1 \alpha, 200 \mathrm{pg} / \mathrm{ml}$; and IL-6, $1 \mathrm{ng} / \mathrm{ml}$ ) with and without THPO (10 ng/ml) for 72 hours. Murine MPL ${ }^{\text {hi }}$ and MPL ${ }^{\text {lo }}$ LTHSCs were cultured in SFEM supplemented with $10 \mathrm{ng} / \mathrm{ml}$ murine IL-3, IL-6, SCF, and Flt3 ligand with and without THPO (10 ng/ml) for 72 hours. Human and murine CML MPL ${ }^{\text {hi }}$ and MPL ${ }^{\text {lo }}$ LTHSCs were also treated with NIL with or without THPO for 72 hours. Cell numbers were measured by Lumino Glo (Promega). For colony-forming capacity assays, the cells were plated in methylcellulose progenitor culture, and burstforming units, erythroid- and colony-forming units, and granulocytes and macrophages were counted after 14 days. To measure apoptosis, cells were labeled with Annexin V (BD) and analyzed by flow cytometry for Annexin V fluorescence. To evaluate in vitro cell cycling, LTHSCs were cultured with EdU (10 $\mu \mathrm{m}$, Invitrogen) for 2 hours, and EdU incorporation was analyzed. For in vivo cell cycling, mice were injected with EdU (Invitrogen, $1 \mathrm{mg}$ per mouse, i.p.). Six hours later, mice were euthanized, MPL ${ }^{\text {hi }}$ and MPL ${ }^{\text {lo }}$ LTHSCs were sorted, and EdU incorporation was analyzed. Cell cycling was also analyzed by anti-Ki-67 (BD) and DAPI labeling, as previously described (46). CD229 (Biolegend) and CD41 (BD) expression on normal and BCR-ABL $\mathrm{MPL}^{\text {hi }}$ and $\mathrm{MPL}^{\text {lo }}$ LTHSCs was analyzed by flow cytometry. LTHSCs were selected from the $\mathrm{BM}$ of nonleukemic and leukemic secondary recipient mice at 8 weeks after transplantation, and p-CRKL (Cell signaling) expression was analyzed by flow cytometry. p-STAT3 and p-STAT5 (BD) expression in murine and human LTHSCs treated with or without THPO (10 $\mathrm{ng} / \mathrm{ml}$ ) was analyzed by flow cytometry. Human CML MPL ${ }^{\text {hi }}$ and MPL ${ }^{\text {lo }}$ LTHSCs were also cultured with and without THPO in the presence of AZD1480 (AstraZeneca, a JAK inhibitor) and PD0325901 (SigmaAldrich, a MEK inhibitor) for 72 hours, and apoptosis, cell cycling, cell growth, and CFC capacity were analyzed as described above. Levels of p-ERK (Ebioscience), p-STAT3, and p-STAT5 were analyzed by flow cytometry analysis. Results are expressed as the ratio of median channel fluorescence of p-CRKL/p-STAT3/5/p-ERK to median channel fluorescence of isotype controls.

Engraftment of murine cells. SCL-tTA/BCR-ABL mice were crossed with GFP-expressing mice to allow tracking of donor cells. Donor $\mathrm{GFP}^{+}$ LTHSCs (200 cells per mouse) were transplanted into a cohort of congenic FVBN mice, which were followed for engraftment of $\mathrm{GFP}^{+}$cells and development of CML. Since the primary recipient mice develop leukemia within 8 weeks and are at risk of becoming sick and dying within this time period, mice were sacrificed at this time point. $\mathrm{GFP}^{+}$LTHSCs were sorted from each primary recipient mouse at 8 weeks after transplant and transplanted into 2 to 3 secondary recipient mice at 200 cells per mouse. Overall, GFP ${ }^{+}$LTHSCs from 6 primary recipient mice were transplanted into 17 secondary recipient mice. For other experiments, CD45.1 MPL ${ }^{\text {hi }}$ and MPL ${ }^{\text {lo }}$ LTHSCs from BCR-ABL mice (200 cells per mouse) or control mice (50 cells per mouse) were sorted and transplanted into CD45.2 congenic recipient mice. Engraftment of donor cells in PB of recipient mice was monitored every 4 weeks and engraftment was monitored in PB, BM, and spleens at 16 weeks. Murine CML and normal LTHSCs (CD45.1) were stained with Hoechst and Pyronin (Sigma-Aldrich), and Hoechst ${ }^{\text {to }}$ Pyronin ${ }^{\text {lo }}$ and Hoechs $\mathrm{t}^{\mathrm{h} / \mathrm{lo}}$ Pyronin ${ }^{\text {hi }}$ MPL hi LTHSCs were selected by flow cytometry and transplanted into CD45.2 recipients (100 cells per mouse).

Gene expression analysis. Total RNA was extracted from LTHSCs using the RNeasy Plus Micro Kit (Qiagen). First-strand cDNA was synthesized from 200 cells using the SuperScript III First-Strand Kit and preamplified for 18 cycles, and Q-RT-PCR analysis was performed by multiplex Q-RT-PCR using TaqMan Gene Expression Assays (Applied Biosystems) and the Biomark HD microfluidic system (Fluidigm). The gene expression assays used are shown in Supplemental Table 3. Results are expressed as a ratio of gene expression to that of $B 2 m$.

RNA deep sequencing with Illumina HiSeq 2500. Sequencing libraries were prepared with the SMARTer Ultra Low Input RNA Kit for Sequencing (v3, TaKaRa Clontech) and the KaPa LTP Library Prep Kit (KaPa Biosystems) following the manufacturer's protocol with minor modifications. Briefly, 500 pg DNA-free RNA from each sample was reverse transcribed and amplified with 13 cycles of Q-RT-PCR using SMART (switching mechanism at 5' end of RNA template) technology. The resulting double-stranded cDNA was sheared with Covaris S220 (Covaris) followed by end repair, 3 '-end adenylation, ligation of bar-coded adapters (Illumina), and 10 cycles of Q-RT-PCR to produce the final sequencing library. Library templates were prepared for sequencing using the cBot cluster generation system with the HiSeq SR Cluster Kit V4 (Illumina). Sequencing was performed using the HiSeq 2500 platform with the HiSeq SBS Kit V4 (Illumina). Real-time analysis 2.2.38 software was used for image analysis and base calling. The Bioconductor ShortRead package was used to analyze FASTQ files, and reads were aligned to the $\mathrm{mm} 9$ reference genome. Analysis of differentially expressed genes was performed using the Bioconductor EdgeR package. Gene function analysis was performed using DAVID Bioinformatics Resources 6.7, and GSEA was performed using GSEA software. The RNA-Seq data has been deposited in the Gene Expression Omnibus database (accession GSE76123).

Lentivirus transduction of murine LTHSCs. MPL shRNA (NM_010823, Sigma-Aldrich) and control shRNA sequences were cloned into the PPIG-U6-GFP plasmid. Lentivirus supernatants were produced and used for transduction of murine LTHSCs as previously described (47). Briefly, BM LTHSCs from BCR-ABL or control mice (CD45.1) were cultured overnight in SFEM medium supplemented with mouse SCF $(10 \mathrm{ng} / \mathrm{ml})$ and mouse THPO (10 ng/ml). The next day, cells were resuspended in SFEM and lentivirus supernatant (multiplicity of infection = 10), supplemented with $10 \mathrm{ng} / \mathrm{ml} \mathrm{SCF}$, and centrifuged at $800 \mathrm{~g}$ for 90 minutes for transduction by spinoculation. For in vitro studies, $\mathrm{GFP}^{+}$cells were selected and analyzed for cell growth, CFC, apoptosis, and cell cycle at 48 hours after transduction. For in vivo transplantation experiments, cells were harvested 24 hours after transduction and injected into irradiated CD45.2 recipient mice (4,000 CML LTHSCs per mouse, 1,000 normal LTHSCs per mouse). Every 4 weeks after transplantation, wbc counts and the percentage of $\mathrm{GFP}^{+}$MPL shRNA-expressing donor cells in the PB were monitored. Engraftment of $\mathrm{GFP}^{+}$donor cells in the $\mathrm{PB}, \mathrm{BM}$, and spleens of the recipient mice was analyzed at 16 weeks after transplantation. At 6 weeks after transplantation, a cohort of mice was euthanized, and apoptosis and cell cycling of $\mathrm{GFP}^{+}$LTHSCs were analyzed. $M p l \mathrm{mRNA}$ expression in $\mathrm{GFP}^{+}$LTHSCs was also analyzed.

Engraftment of human cells in immunodeficient mice. Normal and CML MPL hi and $\mathrm{MPL}^{\text {lo }} \mathrm{Lin}^{-} \mathrm{CD} 34^{+} \mathrm{CD} 38^{-} \mathrm{CD} 90^{+}$cells (CML cells, $3 \times 10^{4}$ cells per mouse for 4 weeks and $6 \times 10^{4}$ cells per mouse for 16 weeks; CB cells, $3 \times 10^{3}$ cells per mouse) were transplanted into irradiated NSG mice (300 cGy, The Jackson Laboratory). Mice were euthanized after 4 or 16 weeks, and BM content of femurs, spleen cells, and blood cells was obtained at necropsy. To assess human cell engraftment, cells were labeled with anti-human CD45 antibody and 
analyzed by flow cytometry. Specific human cell subsets were detected by staining with antibodies to human CD34, CD33, CD14, CD3, and CD19. To assess engraftment of malignant BCR-ABL-expressing cells, human $\mathrm{CD} 45^{+}$cells were selected from BM obtained at 16 weeks after transplantation by immunomagnetic column and evaluated for $B C R$ $A B L$ mRNA levels by Q-RT-PCR.

Statistics. Results are expressed as mean \pm SEM. Comparison between groups was performed by 2-tailed Student's $t$ test or by ANOVA, adjusting for multiple comparisons as appropriate. All statistical analyses were performed using Prism version 5.0 software (GraphPad Software). $P$ values of less than 0.05 were considered to be significant.

Study approval. Mouse care and experimental procedures were performed in accordance with US federal guidelines and protocols approved by the Institutional Animal Care and Use Committee at the Beckman Research Institute of the City of Hope, Duarte, California, USA. Acquisition of human samples was approved by the Institutional Review Boards at the City of Hope, in accordance with the Declaration of Helsinki. All donors signed informed consent forms.

\section{Author contributions}

$\mathrm{BZ}$ designed and conducted experiments and wrote the manuscript. LL conducted experiments. YH conducted experiments. ML analyzed data. GM discussed results and edited the manuscript. WT provided reagents and reviewed the manuscript. RB designed experiments and wrote the manuscript.

\section{Acknowledgments}

This work was supported by NIH NCI grants R01 CA172447 and P30CA033572. We thank StemCyte for CB samples. We thank Linda Seymour and Vincent Paterno for sample acquisition and are grateful for the assistance of the analytical cytometry core and of Chao Guo, Jinhui Wang, and Xiwei Wu at the Integrative Genomics Core at City of Hope.

Address correspondence to: Ravi Bhatia, Division of Hematology and Oncology, Department of Medicine, University of Alabama Birmingham, Birmingham, Alabama 35294, USA. Phone: 205.934.9591; E-mail: rbhatia@uabmc.edu.
1. Sawyers CL. Chronic myeloid leukemia. N Engl J Med.1999;340(17):1330-1340.

2. Ales M, et al. IgG secreting lymphoplasmacytoid leukemia with massive skin involvement and very aggressive clinical course: an exceptionally rare observation. Leuk Res. 2010;34(2):e58-e59.

3. Chu S, et al. Persistence of leukemia stem cells in chronic myelogenous leukemia patients in prolonged remission with imatinib treatment. Blood. 2011;118(20):5565-5572.

4. Mahon FX, et al. Discontinuation of imatinib in patients with chronic myeloid leukaemia who have maintained complete molecular remission for at least 2 years: the prospective, multicentre Stop Imatinib (STIM) trial. Lancet Oncol. 2010;11(11):1029-1035.

5. Ross DM, et al. Patients with chronic myeloid leukemia who maintain a complete molecular response after stopping imatinib treatment have evidence of persistent leukemia by DNA PCR. Leukemia. 2010;24(10):1719-1724.

6. Copley MR, Beer PA, Eaves CJ. Hematopoietic stem cell heterogeneity takes center stage. Cell Stem Cell. 2012;10(6):690-697.

7. Benz C, et al. Hematopoietic stem cell subtypes expand differentially during development and display distinct lymphopoietic programs. Cell Stem Cell. 2012;10(3):273-283.

8. Jamieson CH, et al. Granulocyte-macrophage progenitors as candidate leukemic stem cells in blast-crisis CML. $N$ Engl J Med. 2004;351(7):657-667.

9. Zhang B, et al. Altered microenvironmental regulation of leukemic and normal stem cells in chronic myelogenous leukemia. Cancer Cell. 2012;21(4):577-592.

10. Koschmieder S, et al. Inducible chronic phase of myeloid leukemia with expansion of hematopoietic stem cells in a transgenic model of BCR-ABL leukemogenesis. Blood. 2005;105(1):324-334.

11. Dao L, Morrison L, Kiely G, Zhang C. Spatial distribution of potentially bioavailable metals in surface soils of a contaminated sports ground in Galway, Ireland. Environ Geochem Health. 2013;35(2):227-238.

12. Davies C, et al. Long-term effects of continuing adjuvant tamoxifen to 10 years versus stopping at 5 years after diagnosis of oestrogen receptorpositive breast cancer: ATLAS, a randomised trial. Lancet. 2013;381(9869):805-816.

13. Yoshihara H, et al. Thrombopoietin/MPL signaling regulates hematopoietic stem cell quiescence and interaction with the osteoblastic niche. Cell Stem Cell. 2007;1(6):685-697.

14. Qian H, et al. Critical role of thrombopoietin in maintaining adult quiescent hematopoietic stem cells. Cell Stem Cell. 2007;1(6):671-684.

15. Bersenev A, Wu C, Balcerek J, Tong W. Lnk controls mouse hematopoietic stem cell self-renewal and quiescence through direct interactions with JAK2. JClin Invest. 2008;118(8):2832-2844.

16. Solar GP, et al. Role of c-mpl in early hematopoiesis. Blood. 1998;92(1):4-10.

17. Prost S, et al. Erosion of the chronic myeloid leukaemia stem cell pool by PPAR $\gamma$ agonists. Nature. 2015;525(7569):380-383.

18. Oguro H, Ding L, Morrison SJ. SLAM family markers resolve functionally distinct subpopulations of hematopoietic stem cells and multipotent progenitors. Cell Stem Cell. 2013;13(1):102-116.

19. Sanjuan-Pla A, et al. Platelet-biased stem cells reside at the apex of the haematopoietic stemcell hierarchy. Nature. 2013;502(7470):232-236.

20. Gekas C, Graf T. CD41 expression marks myeloid-biased adult hematopoietic stem cells and increases with age. Blood. 2013;121(22):4463-4472.

21. Cheng T, Rodrigues N, Dombkowski D, Stier S, Scadden DT. Stem cell repopulation efficiency but not pool size is governed by p27(kip1). Nat Med. 2000;6(11):1235-1240.

22. Fatrai S, Wierenga AT, Daenen SM, Vellenga E, Schuringa JJ. Identification of HIF2alpha as an important STAT5 target gene in human hematopoietic stem cells. Blood. 2011;117(12):3320-3330.

23. Fox N, Priestley G, Papayannopoulou T, Kaushansky K. Thrombopoietin expands hematopoietic stem cells after transplantation. JClin Invest. 2002;110(3):389-394.

24. Ohmizono Y, et al. Thrombopoietin augments ex vivo expansion of human cord blood-derived hematopoietic progenitors in combination with stem cell factor and flt3 ligand. Leukemia. 1997;11(4):524-530.

25. Young JC, Bruno E, Luens KM, Wu S, Backer M, Murray LJ. Thrombopoietin stimulates megakaryocytopoiesis, myelopoiesis, and expansion of $\mathrm{CD}_{3} 4^{+}$progenitor cells from single CD34 ${ }^{+}$Thy $-1^{+}$Lin $^{-}$primitive progenitor cells. Blood. 1996;88(5):1619-1631.

26. Matsumoto A, et al. p57 is required for quiescence and maintenance of adult hematopoietic stem cells. Cell Stem Cell. 2011;9(3):262-271.

27. Hantschel O, et al. BCR-ABL uncouples canonical JAK2-STAT5 signaling in chronic myeloid leukemia. Nat Chem Biol. 2012;8(3):285-293.

28. Warsch W, Walz C, Sexl V. JAK of all trades: JAK2-STAT5 as novel therapeutic targets in BCR-ABL1+ chronic myeloid leukemia. Blood. 2013;122(13):2167-2175.

29. Dahlen DD, Broudy VC, Drachman JG. Internalization of the thrombopoietin receptor is regulated by 2 cytoplasmic motifs. Blood. 2003;102(1):102-108.

30. Kilpivaara O, Levine RL. JAK2 and MPL mutations in myeloproliferative neoplasms: discovery and science. Leukemia. 2008;22(10):1813-1817.

31. Heuser M, et al. Modeling the functional heterogeneity of leukemia stem cells: role of STAT5 in leukemia stem cell self-renewal. Blood. 2009;114(19):3983-3993.

32. Cook AM, et al. Role of altered growth factor receptor-mediated JAK2 signaling in growth and maintenance of human acute myeloid leukemia stem cells. Blood. 2014;123(18):2826-2837.

33. Pulikkan JA, et al. Thrombopoietin/MPL participates in initiating and maintaining RUNX1-ETO acute myeloid leukemia via PI3K/AKT signaling. Blood. 2012;120(4):868-879.

34. Chou FS, et al. The thrombopoietin/MPL/Bcl-xL 
pathway is essential for survival and self-renewal in human preleukemia induced by AML1-ETO. Blood. 2012;120(4):709-719.

35. de Laval B, et al. Thrombopoietin-increased DNA-PK-dependent DNA repair limits hematopoietic stem and progenitor cell mutagenesis in response to DNA damage. Cell Stem Cell. 2013;12(1):37-48.

36. Constantinescu SN, Vainchenker W. Small-molecule inhibitors in myeloproliferative neoplasms: are we aiming for the right targets? Hematology Am Soc Hematol Educ Program. 2012;2012:553-560.

37. Gallipoli P, et al. JAK2/STAT5 inhibition by nilotinib with ruxolitinib contributes to the elimination of chronic phase CML CD34 $4^{+}$cells in vitro in vivo. Blood. 2014;124(9):1492-1501.

38. Radich JP, et al. Polymerase chain reaction detection of the BCR-ABL fusion transcript after allogeneic marrow transplantation for chronic myeloid leukemia: results and implications in 346 patients. Blood. 1995;85(9):2632-2638.

39. Mahon FX, et al. Follow-up of complete cytogenetic remission in patients with chronic myeloid leukemia after cessation of interferon alfa. JClin Oncol. 2002;20(1):214-220.

40. Jiang X, et al. Properties of CD $34^{+}$CML stem/ progenitor cells that correlate with different clinical responses to imatinib mesylate. Blood. 2010;116(12):2112-2121.

41. Burchert A. Maintaining low BCR-ABL signaling output to restrict CML progression and enable persistence. Curr Hematol Malig Rep. 2014;9(1):9-16.

42. Bose S, Deininger M, Gora-Tybor J, Goldman JM, Melo JV. The presence of typical and atypical BCR-ABL fusion genes in leukocytes of normal individuals: biologic significance and implications for the assessment of minimal residual disease. Blood. 1998;92(9):3362-3367.
43. Koehrer S, Keating MJ, Wierda WG. Eltrombopag, a second-generation thrombopoietin receptor agonist, for chronic lymphocytic leukemia-associated ITP. Leukemia. 2010;24(5):1096-1098.

44. Olnes MJ, et al. Eltrombopag and improved hematopoiesis in refractory aplastic anemia. NEnglJMed.2012;367(1):11-19.

45. Desmond R, et al. Eltrombopag restores trilineage hematopoiesis in refractory severe aplastic anemia that can be sustained on discontinuation of drug. Blood. 2014;123(12):1818-1825.

46. Jordan CT, Yamasaki G, Minamoto D. High-resolution cell cycle analysis of defined phenotypic subsets within primitive human hematopoietic cell populations. Exp Hematol. 1996;24(11):1347-1355.

47. Modi H,Li L, Chu S, Rossi J, Yee JK, Bhatia R. Inhibition of Grb2 expression demonstrates an important role in BCR-ABL-mediated MAPK activation and transformation of primary human hematopoietic cells. Leukemia. 2011;25(2):305-312. 